TRANSACTIONS OF THE

AMERICAN MATHEMATICAL SOCIETY

Volume 351, Number 12, Pages 4995-5025

S 0002-9947(99)02268-0

Article electronically published on July 21, 1999

\title{
CONFORMAL ITERATED FUNCTION SYSTEMS WITH APPLICATIONS TO THE GEOMETRY OF CONTINUED FRACTIONS
}

\author{
R. DANIEL MAULDIN AND MARIUSZ URBAŃSKI
}

\begin{abstract}
In this paper we obtain some results about general conformal iterated function systems. We obtain a simple characterization of the packing dimension of the limit set of such systems and introduce some special systems which exhibit some interesting behavior. We then apply these results to the set of values of real continued fractions with restricted entries. We pay special attention to the Hausdorff and packing measures of these sets. We also give direct interpretations of these measure theoretic results in terms of the arithmetic density properties of the set of allowed entries.
\end{abstract}

\section{Introduction: Setting And notation}

Let $I$ be a nonempty subset of $\mathbb{N}$, the set of all positive integers such that $I \neq \mathbb{N}$. Let $J_{I}$ be the set of all irrational numbers $z$ whose standard continued fraction has the form

$$
z=\frac{1}{b_{1}+\frac{1}{b_{2}+\frac{1}{b_{3}+\frac{1}{\ddots}}}}
$$

where each partial denominator $b_{i}$ is an element of $I$. We concern ourselves here with the geometric measure theoretic properties of the set $J=J_{I}$. In particular, we are interested in the Hausdorff, packing, and box dimension of $J$ and corresponding measures. It is easy to see (comp. [MU, Section 6]) that $J$ is the limit set of the conformal iterated function system generated by the maps $\phi_{b}(x)=1 /(b+x)$, $b \in I$. Our investigations of $J$ are based on this representation. We call the family $S=\left\{\phi_{b}: b \in I\right\}$ a continued fraction system and $I$ the base for the continued fraction system.

The paper is organized as follows. Later in this section we recall from [MU] some major features of general conformal iterated function systems. In section 2 , we present some new results for general conformal iterated function systems. In particular, we introduce the absolutely regular systems which naturally occur

Received by the editors April 4, 1997.

1991 Mathematics Subject Classification. Primary 28A80; Secondary 58F08, 58F11, 28A78.

Key words and phrases. Iterated function systems, continued fractions, Hausdorff dimension, Hausdorff and packing measures, arithmetic densities.

Research supported by NSF Grant DMS-9502952. 
among the continued fraction systems. For regular systems, we obtain some useful necessary and sufficient conditions for the Hausdorff measure of the limit set $J$ to be positive and also necessary and sufficient conditions for the packing measure to be finite where the dimension parameter for both of these is the Hausdorff dimension of the limit set $J$. We also give a simple and useful characterization of the packing dimension of the limit set in terms of the Hausdorff dimension of $J$ and the box counting dimension of the set of first iterates of a point in the limit set, $J$. In section 4, we apply these results to continued fraction systems. It turns out that when these characterizations are applied to a continued fraction system, these results have direct interpretations in terms of some arithmetic density properties of the set $I$. So, in section 3, we discuss some of these density notions. Some of these notions do not seem to have been discussed before. Again, in section 4, we give the relationship between these density properties and Hausdorff measure and dimension. In section 5 , we give the corresponding properties for packing measure and dimension. In section 6 , we examine some particular continued fraction systems. Various examples are given throughout the paper. The results of this paper include a detailed analysis of those continued fraction systems when the index set $I$ is an arithmetic progression, the set of powers of a given integer, the set of all integers raised to a given exponent, and the set of prime numbers. Finally, we end the paper with some problems which remain unsolved.

Many papers have been written on estimating or determining the Hausdorff dimension of particular sets of continued fractions. The most detailed work has concerned the case where the index set $I$ is finite. We mention here the papers of T.J. Cusick [Cu], I.J. Good [Go], and D. Hensley [He]. However, none of these papers have dealt with the finer geometry of these sets, e.g., whether the Hausdorff measure in the dimension is positive or finite, but have mainly concentrated on other interesting aspects of these finite systems. Also, none of these papers have dealt with the corresponding properties of the packing measure. It is after all a relatively new concept introduced independently by D. Sullivan and C. Tricot in the 1980's. We shall be using several theorems concerning packing measures as presented in Mattila's book [Ma]. If the index set $I$ is finite, then both the Hausdorff and packing measures are positive and finite and each is up to a multiplicative constant the conformal measure corresponding to the system. Here we concentrate on new phenomena which occur when the index set $I$ is infinite. In this paper, we demonstrate that there are many continued fraction systems where the Hausdorff measure is trivial but the packing measure is, geometrically speaking, the correct measure or conversely. We also provide examples for which none of these measures is nontrivial.

We now recall the setting and some of the results developed in [MU] which will be used in this paper. Let $X$ be a nonempty compact subset of a Euclidean space $\mathbb{R}^{d}$. Let $I$ be a countable index set with at least two elements and let $S=$ $\left\{\phi_{i}: X \rightarrow X: i \in I\right\}$ be a collection of injective contractions from $X$ into $X$ for which there exists $0<s<1$ such that $\rho\left(\phi_{i}(x), \phi_{i}(y)\right) \leq s \rho(x, y)$ for every $i \in I$ and for every pair of points $x, y \in X$. Thus, the system $S$ is uniformly contractive. Any such collection $S$ of contractions is called an iterated function system. We are particularly interested in the properties of the limit set defined by such a system. We can define this set as the image of the coding space under a coding map as follows. Let $I^{*}=\bigcup_{n \geq 1} I^{n}$, the space of finite words, and for $\omega \in I^{n}, n \geq 1$, let $\phi_{\omega}=\phi_{\omega_{1}} \circ \phi_{\omega_{2}} \circ \cdots \circ \phi_{\omega_{n}}$. If $\omega \in I^{*} \cup I^{\infty}$ and $n \geq 1$ does not exceed the length of $\omega$, 
we denote by $\left.\omega\right|_{n}$ the word $\omega_{1} \omega_{2} \ldots \omega_{n}$. Since given $\omega \in I^{\infty}$, the diameters of the compact sets $\phi_{\left.\omega\right|_{n}}(X), n \geq 1$, converge to zero and since they form a descending family, the set

$$
\bigcap_{n=0}^{\infty} \phi_{\left.\omega\right|_{n}}(X)
$$

is a singleton and therefore, denoting its only element by $\pi(\omega)$, defines the coding map $\pi: I^{\infty} \rightarrow X$. The main object of our interest will be the limit set

$$
J=\pi\left(I^{\infty}\right)=\bigcup_{\omega \in I^{\infty}} \bigcap_{n=1}^{\infty} \phi_{\omega \mid n}(X) .
$$

Observe that $J$ satisfies the natural invariance equality, $J=\bigcup_{i \in I} \phi_{i}(J)$. Notice that if $I$ is finite, then $J$ is compact. However, our main interest centers on systems $S$ which are infinite. Some of the essential properties of $J$ depend upon an object which appears only when $I$ is infinite. Let $X(\infty)$, the "asymptotic boundary," be the set of limit points of all sequences $\phi_{i}(X), i \in I^{\prime}$, where $I^{\prime}$ ranges over all infinite subsets of $I$. The geometric behavior of the system at $X(\infty)$ directly affects the geometric properties of the limit set $J$. For an infinite continued fraction system the only element of $X(\infty)$ is 0 .

An iterated function system $S=\left\{\phi_{i}: X \rightarrow X: i \in I\right\}$ is said to satisfy the Open Set Condition (abbreviated (OSC)) if there exists a nonempty open set $U \subset X$ (in the topology of X) such that $\phi_{i}(U) \subset U$ for every $i \in I$ and $\phi_{i}(U) \cap \phi_{j}(U)=\emptyset$ for every pair $i, j \in I, i \neq j$.

An iterated function system $S$ satisfying OSC is said to be conformal (c.i.f.s.) if the following conditions are satisfied.

(a) $X$ is a compact connected subset of a Euclidean space $\mathbb{R}^{d}$ and $U=\operatorname{Int}_{\mathbb{R}^{d}}(X)$.

(b) There exist $\alpha, l>0$ such that for every $x \in \partial X \subset \mathbb{R}^{d}$ there exists an open cone $\operatorname{Con}\left(x, u_{x}, \alpha, l\right) \subset \operatorname{Int}(X)$ with vertex $x$, direction vector $u_{x}$, central angle of Lebesgue measure $\alpha$, and altitude $l$.

(c) There exists an open connected set $X \subset V \subset \mathbb{R}^{d}$ such that all maps $\phi_{i}, i \in I$, extend to $C^{1+\varepsilon}$ diffeomorphisms of $V$ into $V$ and are conformal on $V$.

(d) Bounded Distortion Property(BDP). There exists $K \geq 1$ such that $\left|\phi_{\omega}^{\prime}(y)\right| \leq$ $K\left|\phi_{\omega}^{\prime}(x)\right|$ for every $\omega \in I^{*}$ and every pair of points $x, y \in V$, where $\left|\phi_{\omega}^{\prime}(x)\right|$ means the norm of the derivative.

Each continued fraction system $S=\left\{\phi_{b}(x)=1 /(b+x): b \in I\right\}$ satisfies properties (a)-(c). We take $X=[0,1]$. For $V$, we take an open interval such that $X \subset$ $V \subset(-1 / 4,5 / 4)$. To check the bounded distortion property, we note that if $\omega=$ $\left(b_{1}, \ldots, b_{n}\right)$, then $\phi_{\omega}^{\prime}(x)=(-1)^{n} /\left(q_{n}+x q_{n-1}\right)^{2}$. Thus, $\left|\phi_{\omega}^{\prime}(y)\right| \leq 4\left|\phi_{\omega}^{\prime}(x)\right|$, for every pair of points $x, y \in X$. So, we may take the distortion constant $K$ as close to 4 as we like by adjusting the open interval $V$. There is one small point about these continued fraction systems. If $1 \in I$, then the system is not uniformly contractive, since $\phi_{1}^{\prime}(0)=-1$. However, this is not a real problem, since the system of second level maps, $\left\{\phi_{b_{1} b_{2}}: b_{1}, b_{2} \in I\right\}$, has the same limit set and is uniformly contractive.

As was demonstrated in [MU], conformal iterated function systems naturally break into two main classes, irregular and regular. This dichotomy can be determined from either the existence of a zero of a natural pressure function or, equivalently, the existence of a conformal measure. The topological pressure function $P$ 
is defined as follows. For every integer $n \geq 1$ define

$$
\psi_{n}(t)=\sum_{\omega \in I^{n}}\left\|\phi_{\omega}^{\prime}\right\|^{t}
$$

and

$$
P(t)=\lim _{n \rightarrow \infty} \frac{1}{n} \log \psi_{n}(t) .
$$

For a conformal system $S$, we sometimes set $\psi_{S}=\psi_{1}=\psi$. The finiteness parameter, $\theta_{S}$, of the system $S$ is defined by $\inf \{t: \psi(t)<\infty\}=\theta_{S}$. In [MU], it was shown that the topological pressure function $P(t)$ is non-increasing on $[0, \infty)$, strictly decreasing, continuous and convex on $[\theta, \infty)$ and $P(d) \leq 0$. Of course, $P(0)=\infty$ if and only if $I$ is infinite. In [MU] (see Theorem 3.15) we have proved the following characterization of the Hausdorff dimension of the limit set $J$, which will be denoted by $\operatorname{dim}_{H}(J)=h_{S}$.

Theorem 1.1. $\operatorname{dim}_{H}(J)=\sup \left\{\operatorname{dim}_{H}\left(J_{F}\right): F \subset I\right.$ is finite $\}=\inf \{t: P(t) \leq 0\}$. If $P(t)=0$, then $t=\operatorname{dim}_{H}(J)$.

We called the system $S$ regular provided that there is some $t$ such that $P(t)=0$. It follows from [MU] that $t$ is unique. Also, the system is regular if and only if there is a $t$-conformal measure. A Borel probability measure $m$ is said to be $t$-conformal provided $m(J)=1$ and for every Borel set $A \subset X$ and every $i \in I$

$$
m\left(\phi_{i}(A)\right)=\int_{A}\left|\phi_{i}^{\prime}\right|^{t} d m
$$

and

$$
m\left(\phi_{i}(X) \cap \phi_{j}(X)\right)=0,
$$

for every pair $i, j \in I, i \neq j$.

A system $S=\left\{\phi_{i}\right\}_{i \in I}$ is said to be strongly regular if $0<P(t)<\infty$ for some $t \geq 0$. As an immediate application of Theorem 1.1 we get the following

Theorem 1.2. A conformal system $S$ is strongly regular if and only if $h>\theta$.

In [MU] we called a a system $S=\left\{\phi_{i}\right\}_{i \in I}$ hereditarily regular or cofinitely regular provided every nonempty subsystem $S^{\prime}=\left\{\phi_{i}\right\}_{i \in I^{\prime}}$, where $I^{\prime}$ is a cofinite subset of $I$, is regular. A finite system is cofinitely regular and for an infinite system, we showed in $[\mathrm{MU}]$ that whether a system is cofinitely regular can be also determined from the pressure function:

Theorem 1.3. An infinite system $S$ is cofinitely or hereditarily regular if and only if $P(\theta)=\infty \Leftrightarrow \psi(\theta)=\infty \Leftrightarrow\{t: P(t)<\infty\}=(\theta, \infty) \Leftrightarrow\{t: \psi(t)<\infty\}=(\theta, \infty)$.

Theorem 1.4. Every cofinitely regular system is strongly regular.

Note that Example 5.4 of [MU] is a regular, but not strongly regular system. Also, Example 5.3 of [MU] may be adjusted to yield a system $S$ of similarity maps of $[0,1]$ into $[0,1]$ with $\psi_{1}(t)=+\infty$, if $t<1 / 2$ and $\psi_{1}(1 / 2)<1$. Now, add finitely many similarity maps to $S$ so that $\psi_{1}(1 / 2)>1$. This system is strongly, but not cofinitely, regular.

We also need another characterization of the finiteness parameter $\theta$, Theorem 3.23 of $[\mathrm{MU}]$ :

Theorem 1.5. $\theta_{S}=\inf \left\{\operatorname{dim}_{\mathrm{H}}\left(J_{S^{\prime}}\right): S^{\prime}\right.$ is a cofinite subsystem of $\left.S\right\}$. 


\section{ResUlts FOR GENERAL CONFORMAL SYSTEMS}

First, let us introduce another subclass of the regular conformal systems.

Definition. A system $S=\left\{\phi_{i}\right\}_{i \in I}$ is said to be absolutely regular if and only if every nonempty subsystem of $S$ is regular.

The following two statements are obvious.

Theorem 2.1. A system $S$ is absolutely regular if and only if every non-empty subsystem of $S$ is cofinitely regular.

Corollary 2.2. Every absolutely regular system is cofinitely regular.

Like cofinitely regular systems, whether an infinite conformal system is absolutely regular can also be completely determined by the behavior of the pressure function.

Theorem 2.3. Let $S=\left\{\phi_{i}\right\}_{i \in I}$ be infinite. The following conditions are equivalent:

(1) $S$ is absolutely regular.

(2) $\theta_{S}=0$.

Proof. $(2) \Rightarrow(1)$. Assume (2). Then for every infinite subsystem $S^{\prime}, \theta_{S^{\prime}}=0$, and since, $\psi_{S^{\prime}}(0)=\infty, S^{\prime}$ is regular. Thus, $S$ is absolutely regular.

$(1) \Rightarrow(2)$. Suppose $\theta=\theta_{S}>0$. Since $S$ is infinite, we may assume that $I=\mathbb{N}=\{1,2,3, \ldots\}$. Define inductively $I_{n}$, an increasing sequence of finite subsets of $I$, as follows. Set $I_{0}=\emptyset$ and suppose that $I_{n}$ has been determined. Let $M_{n}=\max I_{n}$. Since $\psi\left(\theta\left(1-2^{-n}\right)\right)=\infty$, there exists a finite subset $\Delta_{n}$ of $\left[M_{n}+1, \infty\right)$ such that $\left\|\phi_{i}^{\prime}\right\|^{\theta \cdot 2^{-n}}<2^{-2 n-1}$ for all $i \in \Delta_{n}$ and $2^{n} \leq \sum_{i \in \Delta_{n}}\left\|\phi_{i}^{\prime}\right\|^{\theta\left(1-2^{-n}\right)}$ $\leq 2^{n+1}$. Then by setting $I_{n+1}=I_{n} \cup \Delta_{n}$, we finish the recursion for the sequence $\left\{I_{n}\right\}_{n \geq 0}$. Set $F=\bigcup_{n \geq 0} I_{n}$. By construction, $F$ is infinite. Since $\sum_{i \in \Delta_{n}}\left\|\phi_{i}^{\prime}\right\|^{\theta} \leq$ $2^{-2 n-1} \sum_{i \in \Delta_{n}}\left\|\phi_{i}^{\prime}\right\|^{\theta\left(1-2^{-n}\right)} \leq 2^{-n}$, we conclude that

$$
\psi_{F}(\theta)=\sum_{n \geq 0} \sum_{i \in \Delta_{n}}\left\|\phi_{i}^{\prime}\right\|^{\theta} \leq \sum_{n \geq 0} 2^{-n}=2<\infty
$$

Also for every $0 \leq t<\theta$ there exists $k$ such that $t \leq \theta\left(1-2^{-k}\right)$. Thus,

$$
\psi_{F}(t) \geq \sum_{n \geq k} \sum_{i \in \Delta_{n}}\left\|\phi_{i}^{\prime}\right\|^{t} \geq \sum_{n \geq k} \sum_{i \in \Delta_{n}}\left\|\phi_{i}^{\prime}\right\|^{\theta\left(1-2^{-n}\right)} \geq \sum_{n \geq k} 2^{n}=\infty .
$$

Hence, $\theta_{F}=\theta$ and $\psi_{F}\left(\theta_{F}\right) \leq 2<\infty$. Therefore, the subsystem generated by $F$ is not cofinitely regular and the system $S$ is not absolutely regular.

For a regular conformal system with $P(h)=0$, we know that $\mathcal{H}^{h}(J)<\infty$, where $\mathcal{H}^{h}(J)$ denotes the Hausdorff $h$-dimensional measure. It is possible for the measure to be zero. In Lemma 4.11 of [MU] we gave a sufficient condition for $\mathcal{H}^{h}(J)>0$. In the next theorem we extend this result, by giving some necessary and sufficient conditions for the Hausdorff measure to be positive.

Theorem 2.4. Let $S=\left\{\phi_{i}\right\}_{i \in I}$ be regular. Then the following statements are equivalent.

(1) $\mathcal{H}^{h}(J)>0$. 
$\left(1^{\prime}\right) \exists 0<L<\infty \exists \gamma \geq 1$ such that for all $i \in I$ and for all $r>\gamma \operatorname{diam} \phi_{i}(X)$, there is some $x \in \phi_{i}(X)$ such that

$$
m(B(x, r)) \leq L r^{h} .
$$

(2) $\exists 0<L<\infty \exists \gamma \geq 1$ and there exists a finite set $F$ such that for all $i \in I \backslash F$ and for all $r>\gamma \operatorname{diam} \phi_{i}(X)$, there is some $x \in \phi_{i}(X)$ such that

$$
m(B(x, r)) \leq L r^{h} .
$$

(2') $\exists 0<L<\infty \forall \gamma \geq 1$ there exists a finite set $F$ such that for all $i \in I \backslash F$ and for all $r>\gamma \operatorname{diam} \phi_{i}(X)$, there is some $x \in \phi_{i}(X)$ such that

$$
m(B(x, r)) \leq L r^{h} .
$$

(3) $\exists 0<L<\infty \exists \gamma \geq 1$ and there exists a finite set $F$ such that for all $i \in I \backslash F$ and for all $r>\gamma \operatorname{diam} \phi_{i}(X)$, for all $x \in \phi_{i}(X)$

$$
m(B(x, r)) \leq L r^{h}
$$

(3') $\exists 0<L<\infty \forall \gamma \geq 1$ there exists a finite set $F$ such that for all $i \in I \backslash F$ and for all $r>\gamma \operatorname{diam} \phi_{i}(X)$, for all $x \in \phi_{i}(X)$

$$
m(B(x, r)) \leq L r^{h}
$$

Proof. Obviously $\left(3^{\prime}\right) \Rightarrow(3) \Rightarrow(2) \Rightarrow\left(1^{\prime}\right)$ and $\left(3^{\prime}\right) \Rightarrow\left(2^{\prime}\right) \Rightarrow(2) \Rightarrow\left(1^{\prime}\right)$. It is straightforward to show that $\left(1^{\prime}\right) \Rightarrow(2)$. Lemma 4.11 of $[\mathrm{MU}]$ shows that $\left(1^{\prime}\right) \Rightarrow(1)$. In order to show that (1) implies $\left(3^{\prime}\right)$ suppose that $\left(3^{\prime}\right)$ fails. Then for every $L>$ $1 / \operatorname{dist}^{h}(X, \partial V)$ there exists $j \in I$ such that $m(B(x, r))>L r^{h}$ for some $x \in \phi_{j}(X)$ and some $r>\operatorname{diam}\left(\phi_{j}(X)\right)$. Let $J_{1}$ be the image under $\pi$ of all words of $I^{\infty}$ such that each element of $I$ occurs infinitely often. Consider $z \in J_{1}, z=\pi(\omega) \in I^{\infty}$ such that $\omega_{n+1}=j$ for some $n \geq 1$. Then there exists $z_{n} \in \phi_{j}(X)$ such that $z=\phi_{\left.\omega\right|_{n}}\left(z_{n}\right)$. Since $r \leq 1 / L^{1 / h} \leq \operatorname{dist}(X, \partial V)$, all the geometric consequences of the bounded distortion property listed in Section 2 of [MU] are applicable to the ball $B(x, r)$. In particular, we get $\left|\phi_{\left.\omega\right|_{n}}\left(z_{n}\right)-\phi_{\left.\omega\right|_{n}}(x)\right| \leq D\left\|\phi_{\left.\omega\right|_{n}}^{\prime}\right\| r$ and $B\left(\phi_{\left.\omega\right|_{n}}(x),\left\|\phi_{\left.\omega\right|_{n}}^{\prime}\right\| r\right) \supset$ $\phi_{\left.\omega\right|_{n}}(B(x, r))$. Therefore, $B\left(z,(D+1)\left\|\phi_{\left.\omega\right|_{n}}^{\prime}\right\| r\right) \supset \phi_{\left.\omega\right|_{n}}(B(x, r))$. By conformality and (BDP), this implies that

$$
\begin{aligned}
m\left(B\left(z,(D+1)\left\|\phi_{\left.\omega\right|_{n}}^{\prime}\right\| r\right)\right) & \geq K^{-h}\left\|\phi_{\left.\omega\right|_{n}}^{\prime}\right\|^{h} m(B(x, r)) \geq K^{-h} L\left\|\phi_{\left.\omega\right|_{n}}^{\prime}\right\|^{h} r^{h} \\
& =\left((D+1)\left\|\phi_{\left.\omega\right|_{n}}^{\prime}\right\| r\right)^{h} \frac{L}{K^{h}(D+1)^{h}} .
\end{aligned}
$$

Using Theorem 2.8(1) of [MU], we get $\mathcal{H}^{h}\left(J_{1}\right) \leq C / L$, for some constant $C$ independent of $L$. Now, letting $L \rightarrow \infty$ we conclude that $\mathcal{H}^{h}\left(J_{1}\right)=0$. By Theorem 3.8 and Corollary 3.11 of [MU], $m\left(J \backslash J_{1}\right)=0$. This in turn, in view of Lemma 4.2 of [MU], shows that $\mathcal{H}^{h}\left(J \backslash J_{1}\right)=0$. Thus, $\mathcal{H}^{h}(J)=0$ and therefore $(1) \Rightarrow\left(3^{\prime}\right)$. The proof is finished.

Similarly, we have some necessary and sufficient conditions for the packing measure to be finite. We denote the $h$-dimensional packing measure by $\mathcal{P}^{h}$. For its definition and other information about packing measures and dimensions the reader may look at the book $[\mathrm{Ma}]$ by P. Mattila for example.

Theorem 2.5. Let $S=\left\{\phi_{i}\right\}_{i \in I}$ be regular. Then the following statements are equivalent.

(1) $\mathcal{P}^{h}(J)<\infty$. 
(1') $\exists L>0 \exists \xi>0 \exists \gamma \geq 1$ such that for all $i \in I$ and for all $r$ with $\gamma \operatorname{diam} \phi_{i}(X)<$ $r \leq \xi$ there is some $x \in \phi_{i}(X)$ such that

$$
m(B(x, r)) \geq L r^{h} .
$$

(2) $\exists L>0 \exists \xi>0 \exists \gamma \geq 1$ and there exists a finite set $F$ such that for all $i \in I \backslash F$ and for all $r$ with $\gamma \operatorname{diam} \phi_{i}(X)<r \leq \xi$ there is some $x \in \phi_{i}(X)$ such that

$$
m(B(x, r)) \geq L r^{h} .
$$

(2') $\exists L>0 \exists \xi>0 \forall \gamma \geq 1$ there exists a finite set $F$ such that for all $i \in I \backslash F$ and for all $r$ with $\gamma$ diam $\phi_{i}(X)<r \leq \xi$ there is some $x \in \phi_{i}(X)$ such that

$$
m(B(x, r)) \geq L r^{h} .
$$

(3) $\exists L>0 \exists \xi>0 \exists \gamma \geq 1$ and there exists a finite set $F$ such that for all $i \in I \backslash F$ and for all $x \in \phi_{i}(X)$ and for all $r$ with $\gamma \operatorname{diam} \phi_{i}(X)<r<\xi$

$$
m(B(x, r)) \geq L r^{h} .
$$

(3') $\exists L>0 \exists \xi>0 \forall \gamma \geq 1$ there exists a finite set $F$ such that for all $i \in I \backslash F$ and for all $x \in \phi_{i}(X)$ and for all $r$ with $\gamma \operatorname{diam} \phi_{i}(X)<r<\xi$

$$
m(B(x, r)) \geq L r^{h} .
$$

Proof. It is straightforward to show that $(2) \Rightarrow\left(1^{\prime}\right)$. Lemma 4.10 of $[\mathrm{MU}]$ shows that $\left(1^{\prime}\right) \Rightarrow(1)$. Clearly, $\left(3^{\prime}\right) \Rightarrow(3) \Rightarrow(2)$ and $\left(3^{\prime}\right) \Rightarrow\left(2^{\prime}\right) \Rightarrow(2)$. Finally, by way of contradiction, let us assume (1) holds and ( $\left.3^{\prime}\right)$ fails. Fix $L>0, \xi>0$. Then there are $\gamma \geq 1, i \in I$ and $\gamma \operatorname{diam} \phi_{i}(X)<r \leq \xi$ such that for some $x \in \phi_{i}(X)$, we have

$$
m(B(x, r)) \leq L r^{h} \text {. }
$$

Since the system is regular, there is a Borel subset $B$ of $J$ with $m(B)=1$ and such that each point $\mathrm{z}$ of $B$ has a unique code, $\omega$, and $\pi\left(\sigma^{n}(\omega)\right)$ is in the ball $B(x, r / 2)$ for infinitely many $n$ 's. For such a point $z$ and integer $n \geq 1$, we have

$$
m\left(\phi_{\omega \mid n}\left(B\left(\pi\left(\sigma^{n}(\omega)\right), r / 2\right)\right)\right) \leq\left\|\phi_{\omega \mid n}^{\prime}\right\|^{h} m\left(B\left(\pi\left(\sigma^{n}(\omega)\right), r / 2\right)\right) \leq\left\|\phi_{\omega \mid n}^{\prime}\right\|^{h} L r^{h} .
$$

But, by the bounded distortion property of the system,

$$
\phi_{\omega \mid n}\left(B\left(\pi\left(\sigma^{n}(\omega)\right), r / 2\right)\right) \supset B\left(z,\left\|\phi_{\omega \mid n}^{\prime}\right\| K^{-1} r / 2\right) .
$$

So, $m\left(B\left(z,\left\|\phi_{\omega \mid n}^{\prime}\right\| r / 2 K\right)\right) \leq\left(\left\|\phi_{\omega \mid n}^{\prime}\right\| r / 2 K\right)^{h}(2 K)^{h} L$. Using Theorem 2.9(1) of [MU], we get $\mathcal{P}^{h}(J) \geq \mathcal{P}^{h}(J \cap B) \geq(2 K)^{-h} L^{-1}$. Now, letting $L \rightarrow 0$ we get $\mathcal{P}^{h}(J)=\infty$. This contradiction finishes the proof.

We close this section with a stronger form of Lemma 4.15 of [MU]. This improved version will be directly applied to continued fraction systems in Section 5. For completeness, and to avoid confusion, we have included a proof since part of the hypothesis was unfortunately left out of the statement of Lemma 4.15.

Theorem 2.6. Let $\left\{\phi_{i}: i \in I\right\}$ be a regular conformal iterated function system. Suppose that there exists a subset $\emptyset \neq Z \subset X(\infty)$ such that for every $z \in Z$ there exist $i(z) \in I$ and a set $R(z) \subset(0, \operatorname{dist}(X, \partial V))$ such that

(a) $\phi_{i(z)}(B(z, \sup R(z)) \cap J)=\phi_{i(z)}(B(z, \sup R(z))) \cap J$,

(b) $\phi_{i(z)}(B(z, \sup R(z))) \subset X$,

(c) $\inf \left\{\frac{m(B(z, r))}{r^{h}}: z \in Z, r \in R(z)\right\}=0$.

Then $\mathcal{P}^{h}(J)=\infty$. 
Proof. First notice that since $\varphi_{i(z)}$ is one-to-one, $\phi_{i(z)}(F \cap J)=\phi_{i(z)}(F) \cap J$, for all $z \in Z$ and all $F \subset B(z, \sup R(z))$. Let $J_{\infty}=\pi\left(\Sigma_{\infty}\right)$, where $\Sigma_{\infty} \subset \Sigma$ is the set of all sequences containing each finite word infinitely often. Of course, $J_{\infty}$ has full measure. Fix $\varepsilon>0$ and take $z \in Z$ and $r \in R(z)$ such that $m(B(z, r)) \leq \varepsilon r^{h}$. Fix $x=\pi(\omega), \omega \in \Sigma_{\infty}$. Then there exists $q \geq 1$ such that $\phi_{\omega_{q+1}}(X) \subset B(z, r / 2)$ and $\omega_{q}=i(z)$. Now, $x=\phi_{\left.\omega\right|_{q}}\left(\pi\left(\sigma^{q} \omega\right)\right)$, where $\sigma$ is the shift transformation on the coding space, $I^{N}$. So, using (BDP.3) of [MU], we get

$$
m\left(B\left(x, K^{-1}|| \phi_{\left.\omega\right|_{q}}^{\prime}|| r / 2\right)\right) \leq m\left(\phi_{\left.\omega\right|_{q}}\left(B\left(\pi\left(\sigma^{q} \omega\right), r / 2\right)\right)\right) \leq m\left(\phi_{\left.\omega\right|_{q}}(B(z, r))\right) .
$$

Using the facts that $\phi_{\omega_{q}}(B(z, r)) \subset X$, and condition (a) holds, we have

$$
\begin{aligned}
m\left(\phi_{\left.\omega\right|_{q}}(B(z, r))\right) & =m\left(\phi_{\left.\omega\right|_{q-1}}\left(\phi_{\omega_{q}}(B(z, r))\right)=\int_{\phi_{\omega_{q}}(B(z, r)) \cap J}\left\|\phi_{\left.\omega\right|_{q-1}}^{\prime}\right\|^{h} d m\right. \\
& =\int_{\phi_{\omega_{q}}(B(z, r) \cap J)}\left\|\phi_{\left.\omega\right|_{q-1}}^{\prime}(x)\right\|^{h} d m(x) \\
& =\int_{B(z, r) \cap J}\left\|\phi_{\omega_{q-1}}^{\prime}\left(\phi_{\omega_{q}}(y)\right)\right\|^{h}\left\|\phi_{\left.\omega\right|_{q}}^{\prime}(y)\right\|^{h} d m(y) \\
& =\int_{B(z, r)) \cap J}\left\|\phi_{\left.\omega\right|_{q}}^{\prime}\right\|^{h} d m \leq\left\|\phi_{\omega_{q}}^{\prime}\right\|^{h} m(B(z, r)) \leq\left\|\phi_{\omega_{q}}^{\prime}\right\|^{h} \varepsilon r^{h} \\
& =(2 K)^{-1}\left(\left\|\phi_{\left.\omega\right|_{q}}^{\prime}\right\| r\right)^{h} \varepsilon(2 K)^{h} .
\end{aligned}
$$

Since we may require $q$ to be as large as we wish and since $r>0$ is bounded from above, the numbers $(2 K)^{-1}\left\|\phi_{\left.\omega\right|_{q}}\right\| r$ converge to zero and we finish the proof applying Theorem 2.9(1) of [MU].

Recall that the Hausdorff dimension of a probability measure $m$ is defined by $\operatorname{dim}_{H}(m)=\min \left\{\operatorname{dim}_{H}(E): m(E)=1\right\}$. In Theorem 3.24 of [MU] we have shown that the Hausdorff dimension of the conformal measure of every regular system for which the series $\sum_{i \in I}-\log \left(\left\|\phi_{i}^{\prime}\right\|\right)\left\|\phi_{i}^{\prime}\right\|^{h}$ converges is equal to the Hausdorff dimension of the limit set. In the proof of Corollary 2.25 of [MU] we have demonstrated that this class of systems comprises all the strongly regular systems. Here we shall prove a complementary result for the packing dimension of the conformal measure $m, \operatorname{dim}_{P}(m)=\min \left\{\operatorname{dim}_{P}(E): m(E)=1\right\}$.

Theorem 2.7. If $S$ is a regular system and the series $\sum_{i \in I}-\log \left(\left\|\phi_{i}^{\prime}\right\|\right)\left\|\phi_{i}^{\prime}\right\|^{h}$ converges, then $\operatorname{dim}_{P}(m)=\operatorname{dim}_{H}(J)=\operatorname{dim}_{H}(m)$.

Proof. We shall first show that

$$
\lim _{n \rightarrow \infty} \frac{\log \left(\| \phi_{\left.\omega\right|_{n}}^{\prime}||\right)}{\log \left(\| \phi_{\left.\omega\right|_{n-1}}^{\prime}||\right)}=1
$$

for almost every $\omega \in I^{\infty}$. Indeed, applying Birkhoff's ergodic theorem similarly as it has been done in the beginning of the proof of Theorem 3.24 of [MU], we conclude that for almost every $\omega \in I^{\infty}$ the limit

$$
\lim _{n \rightarrow \infty} \frac{1}{n} \log \left(m\left(\phi_{\omega \mid n}(J)\right)\right)
$$

exists and is independent of $\omega$. Since for all $\omega \in I^{\infty}$ and all $n \geq 1, K^{-h}\left\|\phi_{\left.\omega\right|_{n}}^{\prime}\right\|^{h} \leq$ $m\left(\phi_{\left.\omega\right|_{n}}(J)\right) \leq\left\|\phi_{\left.\omega\right|_{n}}^{\prime}\right\|^{h}$, formula $(2.1)$ is therefore proved. Denote the set of points satisfying (2.1) by $Z$. Fix $\varepsilon>0$. Consider $\omega \in Z$. For $n_{0}$ sufficiently large, we have $\log \left(\| \phi_{\left.\omega\right|_{n}}^{\prime}||\right) \geq(1+\varepsilon) \log \left(\| \phi_{\left.\omega\right|_{n-1}}^{\prime}||\right)$ for all $n \geq n_{0}$ or equivalently $\left\|\phi_{\left.\omega\right|_{n}}^{\prime}\right\| /\left\|\phi_{\left.\omega\right|_{n-1}}^{\prime}\right\| \geq$ 
$\left\|\phi_{\left.\omega\right|_{n-1}}^{\prime}\right\|^{\varepsilon}$. For every $r>0$, let $n$ be the least number satisfying $B(\pi(\omega), r) \supset$ $\phi_{\left.\omega\right|_{n}}(X)$. Then $m(B(\pi(\omega), r)) \geq K^{-h}\left\|\phi_{\left.\omega\right|_{n}}^{\prime}\right\|^{h}$ and $r \leq D\left\|\phi_{\left.\omega\right|_{n-1}}^{\prime}\right\|$, where $D$ is given by (BDP.2) of [MU]. If $r$ is small enough, then $n \geq n_{0}$ and therefore

$$
m(B(\pi(\omega), r)) \geq K^{-h}\left\|\phi_{\left.\omega\right|_{n-1}}^{\prime}\right\|^{h} \cdot \frac{\left\|\phi_{\left.\omega\right|_{n}}^{\prime}\right\|^{h}}{\left\|\phi_{\left.\omega\right|_{n-1}}^{\prime}\right\|^{h}} \geq(D K)^{-h} D^{-h \varepsilon} r^{h+h \varepsilon} .
$$

Thus, $\operatorname{dim}_{P}(\pi(Z)) \leq h+h \varepsilon$ and since $m(Z)=1$, we conclude that $\operatorname{dim}_{P}(m) \leq h$. Since $\operatorname{dim}_{P}(m) \geq \operatorname{dim}_{H}(m)$, the proof is finished.

Finally, we close this section by characterizing the packing dimension of the limit set $J$ of a conformal iterated function system. As recalled in Theorem 1.1, we showed in [MU] that the Hausdorff dimension of $J$ is given by $\operatorname{dim}_{H}(J)=\inf \{t$ : $P(t) \leq 0\}$. It turns out the packing dimension is determined by the box counting dimension of the "level one" portion of the orbits of points of $J$ and the Hausdorff dimension of $J$. For $x \in X, n \in \mathbb{N}$, set $L_{n}(x)=\left\{\phi_{\omega}(x): \omega \in I^{n}\right\}$. We recall that $N_{r}(E)$ is the minimum number of balls of radius $\leq r$ needed to cover a set $E$. We also make some notation. If $F \subset X$ and $R \subset I^{*}$, we denote the set $\bigcup_{\omega \in R} \phi_{\omega}(F)$ by $O(F, R)$. If $R=I^{n}, n \geq 1$, we write $O(F, n)$ for simplicity. In the sequel, we will need the following fact concerning conformal systems. Namely, from (BDP.2) of [MU], there is some number $D>1$ such that

$$
\operatorname{diam}\left(\phi_{\omega}(V)\right) \leq D\left\|\phi_{\omega}^{\prime}\right\|,
$$

for all finite words $\omega$.

Lemma 2.8. Let $\left\{\phi_{i}: i \in I\right\}$ be a conformal iterated function system. Then $\operatorname{dim}_{P}(J)=M=\sup \left\{\operatorname{dim}_{H}(J), \operatorname{dim}_{B}\left(L_{n}(x)\right): x \in J, n \in \mathbb{N}\right\}$.

Proof. Recall from Theorem 3.1 of [MU] that $\operatorname{dim}_{\mathrm{P}}(J)=\overline{\operatorname{dim}}_{\mathrm{B}}(J)$. Fix $t>M$. Since $P(t)<0$, there is some $Q$ such that if $q \geq Q$, then $\psi_{q}(t)<4^{-t}$ and if $|\omega| \geq Q$, then $\left\|\phi_{\omega}^{\prime}\right\| \leq 1 / 4$. Fix $q \geq Q$ and $x \in J$. Choose $A$ such that for all $D \geq r>0, N_{r}\left(L_{q}(x)\right) \leq A r^{-t}$. Now, choose $B$ such that if $1 \leq r \leq D$, then $N_{r}(J) \leq B r^{-t}$ and such that $B \geq 4^{t} A /\left(1-4^{t} \psi_{q}(t)\right)$.

We will show by induction that for each $n \in \mathbb{N}$, if $1 / n \leq r \leq D$, then $N_{r}(J) \leq$ $B r^{-t}$. This inequality holds for 1 . Suppose it holds for $n$ and $1 /(n+1) \leq r<1 / n$. Let $C_{n+1}=\left\{\omega \in I^{q}: \operatorname{diam}\left(\phi_{\omega}(J)\right) \leq 1 / 2(n+1)\right\}$. Since $J=\left(\bigcup_{\omega \in C_{n+1}} \phi_{\omega}(J)\right) \cup$ $\left(\bigcup_{\omega \in I^{q} \backslash C_{n+1}} \phi_{\omega}(J)\right)$, we have

$$
N_{r}(J) \leq N_{1 /(n+1)}(J) \leq N_{1 /(n+1)}\left(\bigcup_{\omega \in C_{n+1}} \phi_{\omega}(J)\right)+\sum_{\omega \in I^{q} \backslash C_{n+1}} N_{1 /(n+1)}\left(\phi_{\omega}(J)\right) .
$$

For $\omega \in I^{q} \backslash C_{n+1}$, we have

$$
N_{1 /(n+1)}\left(\phi_{\omega}(J)\right) \leq N_{1 /\left((n+1)\left\|\phi_{\omega}^{\prime}\right\|\right)}(J) \leq N_{1 /\left(2(n+1)\left\|\phi_{\omega}^{\prime}\right\|\right)}(J) .
$$

Since $\left\|\phi_{\omega}^{\prime}\right\| \leq 1 / 4 \leq(1 / 2)(n / n+1)$, we have $1 / n \leq 1 /\left(2(n+1)\left\|\phi_{\omega}^{\prime}\right\|\right)$. Since $1 /(2(n+1))<\operatorname{diam}\left(\phi_{\omega}(J)\right) \leq D\left\|\phi_{\omega}^{\prime}\right\|$, we have $1 /\left(2(n+1)\left\|\phi_{\omega}^{\prime}\right\|\right) \leq D$. So, by the induction hypothesis, $N_{1 /(n+1)}\left(\phi_{\omega}(J)\right) \leq B\left(2(n+1)\left\|\phi_{\omega}^{\prime}\right\|\right)^{t}$. Next, we claim that $N_{1 /(n+1)}\left(\bigcup_{\omega \in C_{n+1}} \phi_{\omega}(J)\right) \leq N_{1 /(2(n+1))}\left(L_{q}(x)\right)$. To see this, let $B\left(y_{j}, 1 /(2(n+1))\right)$ be a collection of balls of radius $1 /(2(n+1))$ covering $L_{q}(x)$. Suppose $z \in \phi_{\omega}(J)$, where $\omega \in C_{n+1}$. Then $\left|z-\phi_{\omega}(x)\right| \leq \operatorname{diam}\left(\phi_{\omega}(J)\right) \leq 1 /(2(n+1))$. For some 
$j,\left|\phi_{\omega}(x)-y_{j}\right| \leq 1 /(2(n+1))$. So, the balls $B\left(y_{j}, 1 /(n+1)\right)$ cover $\bigcup_{\omega \in C_{n+1}} \phi_{\omega}(J)$. Our claim follows from this. Since $n+1 \leq 2 / r$,

$$
N_{r}(t) \leq A 2^{t}(n+1)^{t}+\sum_{|\omega|=q} B 2^{t}(n+1)^{t}\left\|\phi_{\omega}^{\prime}\right\|^{t} \leq 4^{t}\left[A+B \psi_{q}(t)\right] r^{-t} \leq B r^{-t} .
$$

This completes the induction argument. It now follows that $\overline{\operatorname{dim}}_{\mathrm{B}}(J) \leq t$. From this we have, $\operatorname{dim}_{\mathrm{P}}(J)=M$.

Our goal is to show that we can replace the supremum in Lemma 2.8 with a simple maximum. We use two propositions to accomplish this.

Proposition 2.9. If $S$ is a c.i.f.s., then for all $x, y \in X$, and all $n \geq 1$

$$
\overline{\operatorname{dim}_{B}}(O(x, n))=\overline{\operatorname{dim}_{B}}(O(y, n)) .
$$

Proof. First notice that it suffices to prove this equality for $n=1$ since for every $n \geq 1$ the collection of maps $\left\{\phi_{\omega}: \omega \in I^{n}\right\}$ forms a conformal iterated function system again. With this setting notice that

$$
\exists \geq 1 \forall r>0 \forall z \in R^{d} \#\left\{i \in I: B(z, r) \cap \phi_{i}(X) \neq \emptyset \text { and } \operatorname{diam}\left(\phi_{i}(X)\right) \geq r / 2\right\} \leq M \text {. }
$$

To see this ( $c f$. proof of Lemma 4.11, [MU]) denote the set of such $i$ 's by $E$ and consider $i \in E$ with this property and fix $y \in B(z, r) \cap \phi_{i}(X)$. We repeat here a crucial geometric condition from the definition of a conformal system. The "cone condition" (2.10) of [MU] states: there exists $0<\beta \leq \alpha$ such that for all $x \in X$ and for all $\omega \in I^{*}$

$$
\phi_{\omega}(\operatorname{Int}(X)) \supset \operatorname{Con}\left(\phi_{\omega}(x), \beta, D^{-1}\left\|\phi_{\omega}^{\prime}\right\|\right) \supset \operatorname{Con}\left(\phi_{\omega}(x), \beta, D^{-2} \operatorname{diam}\left(\phi_{\omega}(X)\right)\right),
$$

where $\operatorname{Con}\left(\phi_{\omega}(x), \beta, D^{-1}\left\|\phi_{\omega}^{\prime}\right\|\right)$ and $\operatorname{Con}\left(\phi_{\omega}(x), \beta, D^{-2} \operatorname{diam}(X)\right)$ denote some cones with vertices at $\phi_{\omega}(x)$, angles $\beta$, and altitudes $D^{-1}\left\|\phi_{\omega}^{\prime}\right\|$ and $D^{-2} \operatorname{diam}(X)$ respectively. Thus, there exists a constant $P>0$ such that

$$
\begin{aligned}
\lambda\left(\phi_{i}(X) \cap B(z, 2 r)\right) & \geq \lambda\left(\phi_{i}(X) \cap \operatorname{Con}\left(\phi_{i}(x), \beta, \min \left\{r, D^{-2} \operatorname{diam}\left(\phi_{i}(X)\right)\right\}\right)\right. \\
& \geq \lambda\left(\operatorname{Con}\left(\phi_{i}(x), \beta\left(2 D^{2}\right)^{-1} r\right)\right) \geq \operatorname{Pr}^{2},
\end{aligned}
$$

where $x \in X$ is such that $\phi_{i}(x) \in B(z, 2 r)$. Since all the sets $\phi_{i}(\operatorname{Int}(X)) \cap$ $B(z, 2 r), i \in E$, are mutually disjoint, $\# E \leq \frac{\lambda(B(z, 2 r))}{P r^{2}} \leq \frac{2^{d} V_{d}}{P}$. So, it suffices to take $M=\frac{2^{d} V_{d}}{P}$. In order to prove the proposition, it is enough to show that $\overline{\operatorname{dim}_{\mathrm{B}}}(O(x, 1)) \leq \overline{\operatorname{dim}_{\mathrm{B}}}(O(y, 1))$. Towards this goal, take $0<r \leq \operatorname{diam}(X)$ and let $I_{r}=\left\{i \in I: \operatorname{diam}\left(\phi_{i}(X)\right) \leq r / 2\right\}$. Then $N_{r}\left(O\left(y, I_{r}\right)\right) \leq N_{r / 2}\left(O\left(x, I_{r}\right)\right)$. Clearly, we have $N_{r}\left(O\left(z, I \backslash I_{r}\right)\right) \leq \#\left(I \backslash I_{r}\right)$, for all $z \in X$. On the other hand by (2.2), $N_{r}\left(O\left(z, I \backslash I_{r}\right)\right) \geq \#\left(I \backslash I_{r}\right) / M$. Hence,

$$
\begin{aligned}
N_{r}(O(y, 1)) & \leq N_{r / 2}\left(O\left(x, I_{r}\right)\right)+N_{r}\left(O\left(y, I \backslash I_{r}\right)\right) \\
& \leq N_{r / 2}(O(x, I))+M N_{r}\left(O\left(z, I \backslash I_{r}\right)\right) \leq(1+M) N_{r / 2}(O(x, 1)) .
\end{aligned}
$$

Therefore,

$$
\varlimsup_{r \rightarrow 0} \frac{\log N_{r}(O(y, 1))}{\log r} \leq \varlimsup_{r \rightarrow 0} \frac{\log N_{r}(O(x, 1))}{\log r} .
$$

The proof is finished. 
Lemma 2.10. If $S$ is a c.i.f.s., then for all $x \in X$ and for all $n \geq 1$,

$$
\overline{\operatorname{dim}_{B}}(O(x, n))=\overline{\operatorname{dim}_{B}}(O(x, 1)) .
$$

Proof. By Proposition 2.9, we may assume that $x \in \operatorname{Int}(X)$, so $B(x, \rho) \subset \operatorname{Int}(X)$ for some $\rho>0$. First, we shall show that $\theta_{S} \leq \overline{\operatorname{dim}_{\mathrm{B}}}(O(x, 1))$. To see this, fix $t>t-s>\overline{\operatorname{dim}_{\mathrm{B}}}(O(x, 1))$. Then fix $\varepsilon>0$ and consider the set

$$
I(\varepsilon)=\left\{i \in I: K \varepsilon \rho^{-1} \leq\left\|\phi_{i}^{\prime}(x)\right\| \leq 2 K \varepsilon \rho^{-1}\right\} .
$$

Since the balls $B\left(\phi_{i}(x), \varepsilon\right)$ with $i \in I(\varepsilon)$ are disjoint, $N_{\varepsilon}(O(x, 1)) \geq \# I(\varepsilon)$. Since for all $\varepsilon>0$ small enough $\varepsilon^{-t} \geq \varepsilon^{-s} N_{\varepsilon}(O(x, 1))$, we get for all $k$ large enough, say $k \geq k_{0}$, the following:

$$
\begin{aligned}
\sum_{k \geq k_{0}} \sum_{i \in I\left(2^{-k}\right)}\left\|\phi_{i}^{\prime}\right\|^{t} & \leq \sum_{k \geq k_{0}} 2^{t} K^{t} \rho^{-t} 2^{-k t} \# I\left(2^{-k}\right) \leq 2^{t} K^{t} \rho^{-t} \sum_{k \geq k_{0}} 2^{-k t} N_{2^{-k}}(O(x, 1)) \\
& \leq\left(2 K \rho^{-1}\right)^{t} \sum_{k \geq k_{0}} 2^{-k s} \leq\left(2 K \rho^{-1}\right)^{t} \frac{1}{1-2^{-s}}<\infty
\end{aligned}
$$

Since $\lim _{i \in \mathbb{N}}\left\|\phi_{i}^{\prime}\right\|=0$, the set $I \backslash \bigcup_{k \geq k_{0}} I\left(2^{-k}\right)$ is finite, and therefore $t \geq \theta_{S}$. Letting $t \rightarrow \overline{\operatorname{dim}_{B}}(O(x, 1))$, we get $\theta_{S} \leq \overline{\operatorname{dim}_{B}}(O(x, 1))$.

Now, fix $t>\overline{\operatorname{dim}_{\mathrm{B}}}(O(x, 1))$ again. We shall show by induction that for all $n \geq 1$ there exists $0<A_{n}<\infty$ such that

$$
N_{r}(O(x, n)) \leq A_{n} r^{-t},
$$

for all $0<r \leq 2 D$. Indeed, the existence of $A_{1}$ is immediate as $t>\overline{\operatorname{dim}_{\mathrm{B}}}(O(x, 1))$. Suppose that $0<A_{n}<\infty$ exists. To prove the existence of $A_{n+1}$, set $I_{1}=\left\{\omega \in I^{n}\right.$ : $\left.\operatorname{diam}\left(\phi_{\omega}(X)\right)<r / 2\right\}$. Then $N_{r}\left(O\left(x, I_{1} \times I\right)\right) \leq N_{r / 2}\left(O\left(x, I_{1}\right)\right) \leq N_{r / 2}(O(x, n)) \leq$ $2^{t} A_{n} r^{-t}$. If $\omega \in I^{n} \backslash I_{1}$, then $N_{r}(O(x,\{\omega\} \times I)) \leq N_{r /\left\|\phi_{\omega}^{\prime}\right\|}(O(x, 1)) \leq A_{1}\left\|\phi_{\omega}^{\prime}\right\|^{t} r^{-t}$, where the second inequality sign holds since $r /\left\|\phi_{\omega}^{\prime}\right\| \leq 2 \operatorname{diam}\left(\phi_{\omega}(X)\right) /\left\|\phi_{\omega}^{\prime}\right\| \leq 2 D$. Thus, since $t>\theta_{S}$,

$$
\begin{aligned}
N_{r}(O(x, n+1)) & \leq 2^{t} A_{n} r^{-t}+A_{1} r^{-t} \sum_{\omega \in I^{n} \backslash I_{1}}\left\|\phi_{\omega}^{\prime}\right\|^{t} \leq 2^{t} A_{n} r^{-t}+A_{1} \psi_{n}(t) r^{-t} \\
& =\left(2^{t} A_{n}+A_{1} \psi_{n}(t)\right) r^{-t} .
\end{aligned}
$$

The proof is completed by setting $A_{n+1}=2^{t} A_{n}+A_{1} \psi_{n}(t)$.

As a corollary of Lemma 2.8 and Propositions 2.9 and 2.10, we have a simple means of obtaining the packing dimension of the limit set.

Theorem 2.11. Let $\left\{\phi_{i}: i \in I\right\}$ be a conformal iterated function system. Then $\operatorname{dim}_{P}(J)=\max \left\{\operatorname{dim}_{H}(J), \overline{\operatorname{dim}}_{B}\left(L_{1}(x)\right): x \in J\right\}=\max \left\{\operatorname{dim}_{H}(J), \overline{\operatorname{dim}}_{B}\left(L_{1}\left(x_{0}\right)\right)\right\}$, where $x_{0}$ is any given point in $X$.

\section{Arithmetic Relations}

In this section we collect some basic arithmetic definitions and relations. We begin with the following notation. If $I$ is a subset of $\mathbb{N}$ and $1 \leq p \leq q \leq \infty$ are two real numbers, then by $\# I(p, q)$ we denote the number of elements of the intersection $I \cap[p, q]$. If $p=1$, we frequently use the notation $S_{q}(I)$ for $\# I(p, q)$. 
Lemma 3.1. If $I \subset \mathbb{N}$ and $0<k<l \leq 2 k$, then for all $s>0$

$$
\frac{(k l)^{s}}{(l-k)^{s}} \sum_{n=k}^{l} \frac{1_{I}(n)}{n^{2 s}} \asymp \frac{\# I(k, l)}{(l-k)^{s}},
$$

where the comparability constant depends only on $s$.

Proof. The proof follows immediately from the following computation.

$$
\begin{aligned}
\frac{(k l)^{s}}{(l-k)^{s}} \sum_{n=k}^{l} \frac{1_{I}(n)}{n^{2 s}} & \asymp \frac{l^{2 s}}{(l-k)^{s}} \sum_{n=k}^{l} \frac{1_{I}(n)}{n^{2 s}} \\
& \asymp \frac{l^{2 s}}{(l-k)^{s}} \frac{1}{l^{2 s}} \#(I \cap[k, n])=\frac{\#(I(k, n)}{(l-k)^{s}} .
\end{aligned}
$$

Lemma 3.2. For each $I \subset \mathbb{N}$ and for each $0<t<1$

$$
\varlimsup_{n \rightarrow \infty} \frac{\# I(n / 2, n)}{n^{t}} \geq\left(1-2^{-t}\right) \varlimsup_{n \rightarrow \infty} \frac{\# I(1, n)}{n^{t}} .
$$

Proof. We may assume that $\varlimsup_{n \rightarrow \infty} \frac{\# I(1, n)}{n^{t}}>0$. Let $d=\left(1-2^{-t}\right) \varlimsup_{n \rightarrow \infty} \frac{\# I(1, n)}{n^{t}}$. Suppose on the contrary that $\varlimsup_{k \rightarrow \infty} \frac{\# I\left(2^{k}, 2^{k+1}\right)}{2^{k t}}<d$. Then there exists $c<d$ such that for every $k \geq 1$ large enough, say $k \geq S$, we have $\frac{\# I\left(2^{k}, 2^{k+1}\right)}{2^{k t}} \leq c$. So, for every $k \geq S$,

$$
\frac{\# I\left(2^{S}, 2^{k+1}\right)}{2^{k t}} \leq \sum_{j=S}^{k} \frac{\# I\left(2^{j}, 2^{j+1}\right) \cdot 2^{(j-k) t}}{2^{j t}} \leq \sum_{j=S}^{k} c 2^{(j-k) t} \leq c \frac{1}{1-2^{-t}} .
$$

Thus,

$$
\varlimsup_{k \rightarrow \infty} \frac{\# I\left(1,2^{k+1}\right)}{2^{k t}}=\varlimsup_{k \rightarrow \infty} \frac{\# I\left(2^{S}, 2^{k+1}\right)}{2^{k t}} \leq \frac{c}{1-2^{-t}} .
$$

Now, since for every $n \geq 2$,

$$
\frac{\# I(1, n)}{n^{t}} \leq \frac{\# I\left(1,2^{\left[\log _{2} n\right]+1}\right)}{\left(2^{\left[\log _{2} n\right]}\right)^{t}}
$$

we get

$$
\varlimsup_{n \rightarrow \infty} \frac{\# I(1, n)}{n^{t}} \leq \frac{c}{1-2^{-t}}<\varlimsup_{n \rightarrow \infty} \frac{\# I(1, n)}{n^{t}} .
$$

This contradiction finishes the proof.

We now provide the reader with several definitions of objects and properties associated with infinite subsets of $\mathbb{N}$ which are intended to measure the "size" of those sets.

We first define the lower density dimension of a set $I \subset \mathbb{N}$. Given $t \geq 0$, let

$$
\underline{\varrho}_{t}(I)=\liminf _{l-k \rightarrow \infty}\left\{\frac{\# I(k, l)}{(l-k)^{t}}: k<l \quad \text { and } \quad A\left(\frac{2 k l}{k+l}\right) \cap I \neq \emptyset\right\},
$$

where given $t \geq 0, A(t)=\mathbb{N} \cap[t-1, t+1]$. Notice that

$$
\inf \left\{t: \underline{\varrho}_{t}(I)<\infty\right\}=\sup \left\{t: \underline{\varrho}_{t}(I)>0\right\} .
$$

This common value will be called the lower density dimension of $I$ and will be denoted by $\underline{\varrho} D(I)$. 
Similarly, we define $\varrho \varrho(I)$, the upper density dimension of a set $I \subset \mathbb{N}$, as follows. For each $t \geq 0$, set

$$
\bar{\varrho}_{t}(I)=\limsup _{l-k \rightarrow \infty}\left\{\frac{\# I(k, l)}{(l-k)^{t}}: k<l, k, l \in I\right\}=\limsup _{l-k \rightarrow \infty}\left\{\frac{\# I(k, l)}{(l-k)^{t}}: k<l\right\} .
$$

Notice that

$$
\inf \left\{t: \bar{\varrho}_{t}(I)<\infty\right\}=\sup \left\{t: \bar{\varrho}_{t}(I)>0\right\} .
$$

This common value will be called the upper density dimension of $I$ and will be denoted by $\bar{\varrho} D(I)$. Clearly $\varrho D(I) \leq \bar{\varrho} D(I)$, and if these two numbers are equal, the common value will be denoted by $\varrho D(I)$.

A subset $I \subset \mathbb{N}$ is said to have the strong arithmetic density 0 if for every $t>0$,

$$
\lim _{n \rightarrow \infty} \frac{S_{n}(I)}{n^{t}}=0
$$

We say that two subsets of $\mathbb{N}$ are strongly equivalent if their symmetric difference is finite.

Suppose that $I \subseteq \mathbb{N}$. A subset $A \subset I$ is said to be a cluster of $I$ if and only if $A \cap[\min (A), \sup (A)]=A$. By the length of $A$ we mean the number $\sup (A)-\min (A)$.

A subset $A \subset I$ is said to be a punctured cluster of $I$ if and only if there is $x \notin A$ with $\min (A)<x<\sup (A)$ such that $[\min (A), \sup (A)] \backslash\{x\}=A$. Notice such an $x$ is determined uniquely and by the lower length of $A$ we mean the number $\min \{x-\min (A), \sup (A)-x\}$.

The following lemma, whose straightforward proof is left to the reader, provides some elementary properties of the notions introduced above.

Lemma 3.3. Suppose that $I, I^{\prime} \subseteq \mathbb{N}$. Then

(a) $0 \leq \varrho D(I) \leq \bar{\varrho} D(I) \leq 1$.

(b) If $I^{\prime}$ is strongly equivalent with $I$, then $\varrho D\left(I^{\prime}\right)=\varrho D(I)$ and $\varrho D\left(I^{\prime}\right)=\bar{\varrho} D(I)$.

(c) If I contains arbitrarily long clusters, then $\bar{\varrho} D(I)=1$.

(d) If $\mathbb{N} \backslash I$ contains arbitrarily long punctured clusters, then $\varrho D(I)=0$.

(e) If $p$ is a polynomial of degree $d \geq 1$, then the set $I_{p}=\{[p(n)]: n \in \mathbb{N}\}$ has density dimension $1 / d$.

(f) If I is equivalent with a subset of a geometric sequence, then it has arithmetic density dimension and $\varrho D(I)=0$.

(g) If I is an infinite subset of $\mathbb{N}$ with upper density dimension zero, then I has strong density zero. If in turn I has strong density zero, then it is of lower density dimension zero.

Let us relate the density dimensions of $I$ to the finiteness parameter of the continued fraction system with index set $I$.

Lemma 3.4. If $I \subset \mathbb{N}$, then $\underline{\varrho} D(I) \leq 2 \theta(I) \leq \bar{\varrho} D(I)$.

Proof. Since $I$ is infinite, there exists an infinite subset $F$ of $I$ such that $[n / 2,2 n] \cap$ $[m / 2,2 m]=\emptyset$, for all distinct elements $m$ and $n$ in $F$. Since $\theta_{I} \geq 0$, in order to prove the first inequality we may assume that $\varrho D(I)>0$. Fix then any $0<s<\varrho D(I)$. By the definition of the lower density dimension there exists a constant $M>0$ such that $\# I(k, l) \geq M(l-k)^{s}$, for all $k<l$ with $l-k$ large enough with $A\left(\frac{2 k l}{k+l}\right) \cap I \neq \emptyset$. 
Assuming the first element of $F$ is large enough, we then make the estimates

$$
\begin{aligned}
\psi_{1}(s / 2) & \asymp \sum_{n \in I} \frac{1}{n^{s}} \geq \sum_{n \in F} \sum_{k=\frac{2}{3} n}^{2 n} \frac{1}{k^{s}} 1_{I}(k) \geq \sum_{n \in F} \frac{1}{2^{s}} \cdot \frac{1}{n^{s}} \# I\left(\frac{2}{3} n, 2 n\right) \\
& \geq M \frac{1}{2^{s}} \sum_{n \in F} \frac{1}{n^{s}}\left(\frac{4}{3} n\right)^{s}=\frac{M 2^{s}}{3^{s}} \sum_{n \in F} 1=\infty .
\end{aligned}
$$

Hence, $s / 2 \leq \theta(I)$ and the first inequality is proven. In order to prove the second inequality fix $t>\bar{\varrho} D(I)$ and then auxiliarily $\bar{\varrho} D(I)<s<t$. By the definition of the upper density dimension there exists a constant $0<M<+\infty$ such that $\# I(k, l) \leq M(l-k)^{s}$ for all $k<l$. Then

$$
\begin{aligned}
\psi_{1}(t / 2) & \asymp \sum_{n \geq 0} \sum_{k=2^{n}}^{2^{n+1}} \frac{1}{k^{t}} 1_{I}(k) \asymp \sum_{n \geq 0} \frac{1}{2^{n t}} \# I\left(2^{n}, 2^{n+1}\right) \\
& \leq M \sum_{n \geq 0} \frac{1}{2^{n t}} 2^{n s}=\sum_{n \geq 0} 2^{(s-t) n}<\infty .
\end{aligned}
$$

Hence, $t / 2 \geq \theta(I)$ and we are done.

We end this section with some basic results concerning sets of density zero.

Theorem 3.5. Let $I=\left\{n_{1}<n_{2}<n_{3}<\ldots\right\}$ be an infinite subset of $\mathbb{N}$. The following four statements are equivalent.

(1) I has strong arithmetic density zero.

(2) For each $t>0, \sum_{n \in I} \frac{1}{n^{t}}<\infty$.

(3) For each $t>0, \lim _{k \rightarrow \infty} \frac{k}{n_{k}^{t}}=0$.

(4) The continued fraction system $S=\left\{\phi_{b}\right\}_{b \in I}$ is absolutely regular.

Proof. For each $t>0$, we have by summation by parts

$$
\sum_{k=1}^{n} 1_{I}(k) \frac{1}{k^{t}}=\sum_{k=1}^{n} S_{k}(I)\left[\frac{1}{k^{t}}-\frac{1}{(k+1)^{t}}\right]+\frac{1}{(n+1)^{t}} S_{n}(I) .
$$

So, $\sum_{k=1}^{n} 1_{I}(k) \frac{1}{k^{t}} \leq t \sum_{k=1}^{n} \frac{1}{k} \frac{S_{k}}{k^{t}}+\frac{1}{(n+1)^{t}} S_{n}$. Assume $I$ has strong density zero. Then $M_{t}=\sup _{n \geq 1} \frac{S_{n}(I)}{n^{t}}<\infty, \lim _{n \rightarrow \infty} \frac{S_{n}(I)}{n+1^{t}}=0$, and for all $k, \frac{S_{k}(I)}{k^{t}} \leq \frac{M_{t / 2}}{k^{t / 2}}$. It now follows that $\sum_{k=1}^{n} 1_{I}(k) \frac{1}{k^{t}} \leq t M_{t / 2} \sum_{k=1}^{n} \frac{1}{k^{1+t / 2}}$ and consequently $\sum_{n \in I} \frac{1}{n^{t}}<$ $\infty$. Now, assume statement (2) holds. Then from (3.1), it follows that for each $t>0, \varlimsup \frac{S(n)}{n^{t}}<\infty$. This in turn implies that that for each $t>0, \lim _{n \rightarrow \infty} \frac{S(n)}{n^{t}}=0$. Hence (1) and (2) are equivalent. Now, given $n \geq n_{1}$ take $k$ such that $n_{k} \leq n<$ $n_{k+1}$. Then

$$
\frac{k}{n_{k+1}^{t}} \leq \frac{S_{n}(I)}{n^{t}} \leq \frac{k+1}{n_{k}^{t}}
$$

Thus (3) and (1) are equivalent. Since condition (2) means that $\theta_{I}=0$, the equivalence of (2) and (4) is established by Theorem 2.3. The proof is finished.

One can use the summation by parts formula to obtain another characterization of the finiteness parameter of a continued fraction system: 
Theorem 3.6. Let $I \subset \mathbb{N}$. Then the finiteness parameter $\theta_{I}$ of the continued fraction system with index set I satisfies:

$$
\theta_{I}=\inf \left\{t: \varlimsup_{n \rightarrow \infty} \frac{S_{n}}{n^{t}}<\infty\right\}
$$

The following example completes the part (g) of Lemma 3.3.

Example 3.7. Consider $I=\left\{2^{n}+i: n>0,0 \leq i \leq n-1\right\}$. Then $I$ has positive upper density dimension equal to one and also has strong density zero.

\section{HAUSDORFF MEASURES AND DIMENSIONS}

We begin this section with the following general theorem linking arithmetical properties of the set $I$ and geometrical properties of the corresponding limit set.

Theorem 4.1. For a regular continued fraction system with index set $I$, the following conditions are equivalent:

(a) $\mathcal{H}^{h}\left(J_{I}\right)=0$.

(b) For some $\gamma \geq 1$, $\sup \left\{\frac{m\left(B\left(\phi_{i}(X), r\right)\right)}{r^{h}}: i \in I, r \geq \gamma \operatorname{diam}\left(\varphi_{i}(X)\right)\right\}=\infty$.

(c) For each $\gamma \geq 1, \quad \sup \left\{\frac{m\left(B\left(\phi_{i}(X), r\right)\right)}{r^{h}}: i \in I, r \geq \gamma \operatorname{diam}\left(\varphi_{i}(X)\right)\right\}=\infty$.

(d) $\sup _{k<l} \frac{(k l)^{h}}{(l-k)^{h}} \sum_{n=k}^{l} \frac{1_{I}(n)}{n^{2 h}}=\infty$.

Proof. First, let us assume statement (a) holds and fix $\gamma \geq 1$. Since statement (2) of Theorem 2.4 is false, the ratios $m(B(x, r)) / r^{h}$ with $x \in \phi_{i}(X)$ and $r>$ $\gamma \operatorname{diam}(\phi(X))$ can be made arbitrarily large. Thus, (a) $\Rightarrow(\mathrm{b})$. Clearly, (c) $\Rightarrow(\mathrm{b})$.

Next, let us assume $\gamma_{0} \geq 1$ is such that the supremum in statement (b) is $\infty$. Let $L>0$. We will show that condition $\left(3^{\prime}\right)$ of Theorem 2.4 fails with $\gamma=$ $\gamma_{0}+1$. First, note that if $j \in I$ and $r \geq \gamma \operatorname{diam}\left(\varphi_{j}(X)\right)$, then $m\left(B\left(\phi_{j}(X), r\right)\right) / r^{h} \leq$ $\left(\gamma \operatorname{diam}\left(\phi_{j}(X)\right)\right)^{-h}$. Let $F \subset I$ be finite. Let

$$
T=\max \left\{\gamma^{h} L,\left(\gamma \operatorname{diam} \varphi_{j}(X)\right)^{-h}: j \in F\right\} .
$$

Choose $i \in I$ and $r_{0} \geq \gamma_{0} \operatorname{diam}\left(\varphi_{i}(X)\right)$ such that $m\left(B\left(\phi_{i}(X), r_{0}\right)\right) \geq(T+1) r_{0}^{h}$. Thus, $i \in I \backslash F$. Let $x \in \varphi_{i}(X)$ and $r=\left(1+1 / \gamma_{0}\right) r_{0}$. Then $r \geq \gamma \operatorname{diam} \varphi_{i}(X)$ and $B(x, r) \supset B\left(\varphi_{i}(X), r_{0}\right)$. So, $m(B(x, r))>L r^{h}$. Thus, by Theorem $2.4, \mathcal{H}^{h}\left(J_{I}\right)=0$. So, (b) $\Rightarrow(\mathrm{a})$.

Now, assume statement (d) holds. We show that statement (b) holds with $\gamma=1$. Choose $k<l$ such that $\frac{(k l)^{h}}{(l-k)^{h}} \sum_{n=k}^{l} \frac{1_{I}(n)}{n^{2 h}}>T>0$, where a positive lower bound on $T$ will be specified later. Choose $i \in I$ such that the distance from $1 / i$ to $1 / 2(1 / k+1 / l)$ is minimum and let $r=\max \{|1 /(i+1)-1 /(l+1)|,|1 / k-1 / i|\}$. So, $r<1 / k-1 / l$ and

$$
\frac{m\left(B\left(\varphi_{i}(X), r\right)\right)}{r^{h}} \geq\left(K^{-h}\right) \frac{(k l)^{h}}{(l-k)^{h}} \sum_{n=k}^{l} \frac{1_{I}(n)}{n^{2 h}} \geq K^{-h} T .
$$

Let us note that the quantities $\frac{(k l)^{h}}{(l-k)^{h}} \sum_{n=k}^{l} \frac{1_{I}(n)}{n^{2 h}}$ with $r<\operatorname{diam}\left(\phi_{i}(X)\right)=$ $1 / i(i+1)$, where $r$ is chosen as above have a uniform upper bound. To see this 
note that in this setting $k=i$ and $l<i(i+1) /(i-1)$. So, $\frac{(k l)^{h}}{(l-k)^{h}} \sum_{n=k}^{l} \frac{1_{I}(n)}{n^{2 h}}$ is bounded above by $\frac{(k l)^{h}}{(l-k)^{h}} \frac{1}{i^{2 h}} \frac{2 i}{(i-1)}$. If $l \leq 2 k$, then $\frac{(k l)^{h}}{(l-k)^{h}} \leq(k l)^{h}$ and using the bounds on $l$, we get the quantity uniformly bounded above. If $2 k<l$, then $\frac{(k l)^{h}}{(l-k)^{h}} \leq\left(\frac{i(i+1)}{i-1}\right)^{h}$, and again the quantity is uniformly bounded above. Therefore, we also have $r \geq \operatorname{diam}\left(\phi_{i}(X)\right)$, for $T$ sufficiently large. Thus, $(\mathrm{d}) \Rightarrow(\mathrm{b})$.

Finally, let us assume statement (c) holds and take $\gamma=1$. Let $m\left(B\left(\phi_{i}(X), r\right)\right) / r^{h}$ $>T$, with $r \geq \operatorname{diam}\left(\phi_{i}(X)\right)$. Choose $k<l$ such that $[1 /(l-1), 1 / k+1] \subset$ $B\left(\phi_{i}(X), r\right)$ and $1 / k, 1 / l \notin B\left(\phi_{i}(X), r\right)$. Then $1 / k-1 / l \leq 4 r$ and

$$
\frac{(k l)^{h}}{(l-k)^{h}} \sum_{n=k}^{l} \frac{1_{I}(n)}{n^{2 h}} \geq\left(4^{-h}\right) \frac{m\left(B\left(\phi_{i}(X), r\right)\right)}{r^{h}} .
$$

From this, we see $(c) \Rightarrow(d)$.

Corollary 4.2. If $I \subseteq \mathbb{N}$ generates a regular continued fraction system and if

$$
\varlimsup_{n \rightarrow \infty} \frac{\# I(1, n)}{n^{h}}=\infty,
$$

then $\mathcal{H}^{h}\left(J_{I}\right)=0$.

Proof. For every $k \geq 1$ we have

$$
\frac{(k \cdot 2 k)^{h}}{(2 k-k)^{h}} \sum_{n=k}^{2 k} \frac{1_{I}(n)}{n^{2 h}}=2^{h} k^{h} \sum_{n=k}^{2 k} \frac{1_{I}(n)}{n^{2 h}} \geq 2^{h} k^{h} \frac{\# I(k, 2 k)}{(2 k)^{2 h}}=2^{-h} \frac{\# I(k, 2 k)}{k^{h}} .
$$

Thus an immediate application of Theorem 4.1 and Lemma 3.2 finishes the proof.

As an immediate consequence of this result we get the following.

Corollary 4.3. Let $I$ be a base for a regular continued fraction system and let $h$ be the dimension of the system. If for some $t>h$, $\limsup _{n \rightarrow \infty} \frac{\# I(1, n)}{n^{t}}>0$, then $\mathcal{H}^{h}(J)=0$.

Proposition 4.4. If $I$ is a base for a continued fraction system and $\mathcal{H}^{h}\left(J_{I}\right)>0$, then $h \geq 2 \theta$.

Proof. Since the Hausdorff measure is a conformal measure, the system is regular. By Corollary 4.2, $\quad M=\sup _{n} \frac{\# I(1, n)}{n^{h}}<\infty$. Suppose by way of contradiction that $h<2 \theta$. Thus $\theta>0$ and there exists $0<t<\theta$ such that $h<2 t$. Then we can write

$$
\begin{aligned}
\psi_{1}(t) & =\sum_{n \in I} \frac{1}{n^{2 t}}=\sum_{n=0}^{\infty} \sum_{k \in I \cap\left[2^{n}, 2^{n+1}\right)} \frac{1}{k^{2 t}} \leq \sum_{n=0}^{\infty} \frac{\# I\left[2^{n}, 2^{n+1}\right)}{2^{2 t n}} \\
& =\sum_{n=0}^{\infty} \frac{\# I\left[2^{n}, 2^{n+1}\right)}{2^{n h}} 2^{(h-2 t) n} \leq \frac{2^{h} M}{1-2^{-(2 t-h)}}<\infty .
\end{aligned}
$$

Hence, $\psi_{1}(t)$ is finite which contradicts the definition of $\theta$ and finishes the proof.

Since by Theorem 4.7 of $[\mathrm{MU}], h<1$ if $I \neq \mathbb{N}$, as an immediate consequence of this proposition, we get the following 
Corollary 4.5. If $I \neq N$ is a base for a continued fraction system and $\mathcal{H}^{h}\left(J_{I}\right)>0$, then $\theta<1 / 2$. In particular, if $I$ is the set of all prime numbers, then $\mathcal{H}^{h}\left(J_{I}\right)=0$.

Proof. Since $p_{n} \asymp n \log n$, it easily follows that if $I$ is the set primes, then $\theta_{I}=1 / 2$, $\psi_{I}(1 / 2)=\infty$ and the system is strongly regular.

Let us also note the following, in a sense stronger, consequence of Proposition 4.4.

Corollary 4.6. Let the infinite set $I$ be the base for a continued fraction system. If $\mathcal{H}^{h_{F}}\left(J_{F}\right)>0$, for every cofinite subsystem $F$ of $I$, then I has strong density 0.

Proof. Since the Hausdorff measure is a conformal measure, the system is regular. Suppose that $I$ does not have strong density 0 . Therefore by Theorem 2.3 and Theorem 3.5, $\theta(I)>0$. Thus, applying Theorem 1.5 and Lemma 3.19 of [MU] we see that there is a cofinite subsystem $F$ of $I$ such that $h_{F}<2 \theta(F)$. This contradicts Proposition 4.4 and finishes the proof.

Lemma 4.7. Suppose $I \subset \mathbb{N}, I \neq \mathbb{N}$ and $I$ contains arbitrarily long blocks; then $\mathcal{H}^{h}\left(J_{I}\right)=0$.

Proof. By way of contradiction, suppose $\mathcal{H}^{h}\left(J_{I}\right)>0$. By Theorem 4.1.7 of [MU], the system is regular. If $I$ has a block from $k$ to $l$, then

$$
\frac{(k l)^{h}}{(l-k)^{h}} \sum_{n=k}^{l} \frac{1_{I}(n)}{n^{2 h}}=\frac{(k l)^{h}}{(l-k)^{h}} \sum_{n=k}^{l} \frac{1}{n^{2 h}} \geq \frac{(k l)^{h}}{(l-k)^{h}} \frac{(l-k)}{l^{2 h}}=\left(\frac{k}{l}\right)^{h}(l-k)^{1-h} .
$$

If additionally $l \leq 2 k$, then $\left(\frac{k}{l}\right)^{h}(l-k)^{1-h} \geq 2^{-h}(l-k)^{1-h}$. Since $h<1$ and since $I$ has arbitrarily long blocks $(l-k \rightarrow \infty)$ with the property that $l \leq 2 k$, we complete the proof by invoking Theorem 4.1.

Lemma 4.8. If $I \subseteq \mathbb{N}$ and $h=\operatorname{dim}_{H}\left(J_{I}\right)>\bar{\varrho} D(I)$, then $\mathcal{H}^{h}\left(J_{I}\right)>0$.

Proof. Since $h>\bar{\varrho} D(I)$, there exists a constant $M>0$ such that $\# I(k, l) \leq$ $M(l-k)^{h}$ for all $0<k<l$. Thus, it follows from Lemma 3.1 that

$$
\sup _{k<l \leq 2 k} \frac{(k l)^{h}}{(l-k)^{h}} \sum_{n=k}^{l} \frac{1_{I}(h)}{n^{2 h}}<\infty .
$$

If $l>2 k>0$, then $\frac{(k l)^{h}}{(l-k)^{h}} \asymp \frac{k^{h} l^{h}}{l^{h}}=k^{h}$, and therefore, for $l>2 k$,

$$
\begin{aligned}
\frac{(k l)^{h}}{(l-k)^{h}} \sum_{n=k}^{l} \frac{1_{I}(h)}{n^{2 h}} & \asymp k^{h} \sum_{n=k}^{l} \frac{1_{I}(h)}{n^{2 h}} \leq k^{h} \sum_{n=k}^{\infty} \frac{1_{I}(h)}{n^{2 h}} \\
& =k^{h} \sum_{j=0}^{\infty} \sum_{n=2^{j} k}^{2^{j+1} k-1} \frac{1_{I}(h)}{n^{2 h}} \leq k^{h} \sum_{j=0}^{\infty} \frac{\#\left(I \cap\left[2^{j} k, 2^{j+1} k\right]\right)}{\left(2^{j} k\right)^{2 h}} \\
& =\frac{1}{k^{h}} \sum_{j=0}^{\infty} \frac{\#\left(I \cap\left[2^{j} k, 2^{j+1} k\right]\right)}{2^{j 2 h}} \leq \frac{M}{k^{h}} \sum_{j=0}^{\infty} \frac{\left(2^{j} k\right)^{h}}{2^{2 j h}} \\
& =M \sum_{j=0}^{\infty} \frac{1}{2^{j h}}=\frac{M}{1-2^{-h}}<\infty .
\end{aligned}
$$

By Lemma 3.4, $h>\bar{\varrho} D(I) \geq 2 \theta \geq \theta$ and, therefore, by Theorem 1.2, $I$ induces a regular system. Thus, an application of Theorem 4.1 finishes the proof. 
Corollary 4.9. If I is strongly equivalent to $\left\{a^{n}: n \in \mathbb{N}\right\}$, for some $a \in \mathbb{N}, a \geq 2$, then $\mathcal{H}^{h}\left(J_{I}\right)>0$.

Remark. As it follows from the proof of Lemma 4.8, it suffices to require the existence of a constant $M>0$ such that $\# I(k, l) \leq M(l-k)^{h}$ for all $0<k<l$ which is weaker than the assumption $h=\operatorname{dim}_{\mathrm{H}}\left(J_{I}\right)>\bar{\varrho} D(I)$.

Remark. Since there exist absolutely regular systems $I$ with arbitrarily long blocks, it is possible to have $\mathcal{H}^{h}\left(J_{I}\right)=0$ for an absolutely regular system.

Theorem 4.10. If $\varrho D(I)<1$, then the strong equivalence class of I contains an element $F$ with $\mathcal{H}^{h_{F}}\left(J_{F}\right)>0$. More precisely, there exists a number $q \geq 1$ such that if $F$ is strongly equivalent with $I$ and $F \supseteq I \cup[1, q]$, then $\mathcal{H}^{h_{F}}\left(J_{F}\right)>0$.

Proof. In view of Theorem 1.1 there exists $q \geq 1$ such that $\operatorname{dim}_{\mathrm{H}}\left(J_{F}\right)>\bar{\varrho} D(I)$ provided $F \supseteq[1, q]$. So, we finish the proof applying Lemma 4.8.

Remarks. Notice that combining Theorem 4.10 and Lemma 3.3(e) and (f) gives rise to a method of producing a large class of sets $I$ with $\mathcal{H}^{h_{I}}\left(J_{I}\right)>0$. We also note that the property of being cofinitely regular is invariant under strong equivalency whereas regularity is not.

Lemma 4.11. Let $I \subset \mathbb{N}, k \geq e^{2}$, and $l \geq k$. The function $g_{k, l}$ defined by $t \mapsto$ $\frac{(k l)^{t}}{(l-k)^{t}} \sum_{n=k}^{l} \frac{1_{I}(n)}{n^{2 t}}$ is non-increasing.

Proof. One simply calculates

$$
g_{k, l}^{\prime}(t)=\frac{(k l)^{t}}{(l-k)^{t}}\left(\ln \left(\frac{k l}{l-k}\right)-2\right) \sum_{n=k}^{l} \frac{1_{I}(n)(1-\ln n)}{n^{2 t}} .
$$

If $k \geq e^{2}$, then $\ln \left(\frac{k l}{l-k}\right)-2 \geq 0$ and $1-\ln n<0$ for all $n \geq k \geq e^{2}$. Thus, $g_{k, l}^{\prime}(t) \leq 0$ and we are done.

One may generalize Proposition 4.4:

Lemma 4.12. Let $I \subset \mathbb{N}$. If $\frac{(k l)^{s}}{(l-k)^{s}} \sum_{n=k}^{l} \frac{1_{I}(n)}{n^{2 s}}<M<\infty$, for some $s \geq 0$, then $s \geq 2 \theta$.

Proof. Fix $t>s / 2$. Then

$$
\begin{aligned}
\psi_{1}(t) & =\sum_{n=0}^{\infty} \sum_{j=2^{n}}^{2^{n+1}} \frac{1_{I}(j)}{j^{2 s} j^{2(t-s)}} \leq \sum_{n=0}^{\infty} 2^{2 n(s-t)} \sum_{j=2^{n}}^{2^{n+1}} \frac{1_{I}(j)}{j^{2 s}} \leq 2^{-s} \sum_{n=0}^{\infty} 2^{2 n(s-t)} \cdot M 2^{-n s} \\
& \leq \frac{2^{-s} M}{1-2^{s-2 t}}<\infty .
\end{aligned}
$$

Thus, $t \geq \theta$ and letting $t \searrow s / 2$, we get $s / 2 \geq \theta$.

Theorem 4.13. If $I \subset \mathbb{N}$ and $\mathcal{H}^{h_{I}}\left(J_{I}\right)>0$, then $\mathcal{H}^{h_{E}}\left(J_{E}\right)>0$, for every system $E \supset I$ such that $E \backslash I$ is finite.

Proof. As $h_{E} \geq h_{I}$, it follows from Theorems 4.1 and 4.11 that

$$
\sup _{k \geq 1} k^{-h_{E}} \sum_{n \geq k} \frac{1_{I}(n)}{n^{2 h_{E}}}<\infty .
$$

Since $\sum_{n \geq k} \frac{1_{E}(n)}{n^{2 h} E}=\sum_{n \geq k} \frac{1_{I}(n)}{n^{2 h} E}$, for $\mathrm{k}$ large enough, $\sup _{k \geq 1} k^{-h_{E}} \sum_{n \geq k} \frac{1_{E}(n)}{n^{2 h} E}<$ $\infty$. Also, the system $E$ is regular, since every system containing a regular system as a cofinite subset is regular. Invoking Theorem 4.1 again, we get $\mathcal{H}^{h_{E}}\left(J_{E}\right)>0$. 


\section{PACKIng MEASURES AND Dimensions}

We begin this section by giving some necessary and sufficient conditions for the packing measure to be finite for regular systems. Since the packing measure is more complex than Hausdorff measure, we must analyze separately those index sets $I$ which are cofinite and those which are not. It is in the proof of Theorem 5.1 that the use of the harmonic mean of $k$ and $l, H(k, l)$, in connection with packing measure becomes essential.

Theorem 5.1. For a regular continued fraction system with index set $I$, the following three conditions are equivalent:

(a) $\mathcal{P}^{h}(J)<\infty$.

(b)

$$
\inf _{\substack{k<l \\ A\left(\frac{2 k l}{k+l}\right) \cap I \neq \emptyset}} \frac{(k l)^{h}}{(l-k)^{h}} \sum_{n=k}^{l} \frac{1_{I}(n)}{n^{2 h}}>0 \quad \text { and } \quad \inf _{1 \leq k} k^{h} \sum_{n=k}^{\infty} \frac{1_{I}(n)}{n^{2 h}}>0 .
$$

(c) For some $k_{0}, n_{0}$,

$$
\inf _{\substack{k_{0}<k, k+n_{0}<l \\ A\left(\frac{2 k l}{k+l}\right) \cap I \neq \emptyset}} \frac{(k l)^{h}}{(l-k)^{h}} \sum_{n=k}^{l} \frac{1_{I}(n)}{n^{2 h}}>0 \text { and } \inf _{1 \leq k} k^{h} \sum_{n=k}^{\infty} \frac{1_{I}(n)}{n^{2 h}}>0 .
$$

Proof. Clearly, $(\mathrm{b}) \Rightarrow(\mathrm{c})$. Suppose (c) holds for some given $k_{0}, n_{0}$. Since the quantities in the first infimum in (b) are uniformly bounded away from 0 if $l-k \leq n_{0}$, we need consider only the quantities where $k \leq k_{0}$ and $l>k+n_{0}$. If the infimum of these quantities is 0 , then there are some fixed $k \leq k_{0}$ and an infinite sequence of $l$ 's such that the quantities converge to 0 . But, the limit of this sequence of quantities is $k^{h} \times\left(\psi_{1}(h)-\sum_{n=1}^{k-1} \frac{1_{I}(n)}{n^{2 h}}\right)$ which is positive. Thus, $(\mathrm{c}) \Rightarrow(\mathrm{b})$.

Now, suppose that condition (a) is satisfied. We will show that the first inequality in (c) holds with $k_{0}=6$ and $n_{0}$ large enough (we will indicate that $n_{0} \leq 49$ suffices). Consider $k+49<l, k>6$ and such that $A\left(\frac{2 k l}{k+l}\right) \cap I \neq \emptyset$. Let $i \in I$ be a point closest to $2 k l /(k+l)$. Then $k<i<l$ and there exists $x \in\left(\frac{1}{i+1}, \frac{1}{i}\right) \cap J_{I}$. Set $r=\min \{1 / k-x, x-1 / l\}$. It can be shown under these conditions that the inequalities $1 /(i+1)-1 / l>1 / i(i+1)$ and $1 / k-1 / i>1 / i(i+1)$ hold. It now follows that $r>\operatorname{diam}\left(\varphi_{i}(X)\right)$. Also, $\frac{1}{l+1} \leq x-r<x+r \leq \frac{1}{k}$ and therefore

$$
B(x, r) \cap J_{I} \subset\left[\frac{1}{l+1}, \frac{1}{k}\right] \cap J_{I}=\bigcup_{j \in[k, l] \cap I}\left[\frac{1}{j+1}, \frac{1}{j}\right] \cap J_{I} .
$$

It also follows from the conditions on $k$ and $l$ that the following inequalities hold: $1 / k-1 / l<4(1 / k-1 / i)$ and $1 / k-1 / l<4(1 / i+1-1 / l)$. From this we get $1 / k-1 / l<4 r$. Since condition $\left(2^{\prime}\right)$ of Theorem 2.5 with $\gamma=1$ holds, we find there is a positive number $L$ such that

$$
\frac{(k l)^{h}}{(l-k)^{h}} \sum_{n=k}^{l} \frac{1_{I}(n)}{n^{2 h}} \geq 4^{-h} \frac{m(B(x, r))}{r^{h}} \geq L .
$$

So, the first infimum in (c) is positive. To see that the second infimum is positive, note that for each $k, \frac{m(B(0,1 / k))}{1 / k^{h}} \asymp k^{h} \sum_{n=k}^{l} \frac{1_{I}(n)}{n^{2 h}}$. Consider two cases. First, suppose that there exists $2 \leq j \in I$ such that $j-1 \notin I$. If the infimum is zero, then the assumptions of Theorem 2.6 are satisfied with $Z=\{0\}, i(0)=j$, 
and $R(0)=\{1 / k: k \geq 4\}$. Hence, $\mathcal{P}^{h}\left(J_{I}\right)=\infty$ and we have a contradiction. Otherwise, $I=\mathbb{N}$ or $I=\mathbb{N} \backslash\{1\}$. Then $\theta=1 / 2$ and for all $k \geq 2$

$$
k^{h} \sum_{n=k}^{\infty} \frac{1_{I}(n)}{n^{2 h}}=k^{h} \sum_{n=k}^{\infty} \frac{1}{n^{2 h}} \asymp k^{h} k^{1-2 h}=k^{1-h} .
$$

Since $\lim _{k \rightarrow \infty} k^{1-h}=\infty$, our infimum is also positive in this case.

Finally, suppose that condition (b) is satisfied. We will show condition (2) of Theorem 2.5 holds. Let $M>0$ be the first infimum appearing in (b). Fix $\gamma \geq 1$ which will be specified later on. Consider $i \in I$ and $\gamma / i(i+1)<r<1 / i$. Set $k=\left[1 /\left(\frac{1}{i}+r\right)\right]+1$ and $l=\left[1 /\left(\frac{1}{i}-r\right)\right]-1$. Then $\frac{1}{l+2}+\frac{1}{k}<\frac{2}{i}<\frac{1}{k-1}+\frac{1}{l+1}$ which equivalently means that $H(k-1, l+1) \leq i \leq H(k, l+2)$, where $H(a, b)=2 a b / a+b$, the harmonic mean of $a$ and $b$. Since $H(k-1, l+1) \leq H(k, l+1) \leq H(k, l+2)$ and $H(k, l+2)<H(k, l+1)+1<H(k-1, l+1)+2$, there exists $(a, b) \in$ $\{(k-1, l+1),(k, l+1),(k, l+2)\}$ such that $|H(a, b)-i| \leq 1$ which means that $i \in A(a, b) \cap I$. Moreover, $r \leq \frac{1}{2}\left(\frac{1}{k-1}-\frac{1}{l+2}\right) \leq 4\left(\frac{1}{a}-\frac{1}{b}\right)$. Choose $x \in J \cap(1 / i+1,1 / i)$ so close to $1 / i$ that $B(x, r) \supset \bigcup_{j=k}^{l-1}[1 / j+1,1 / j]$. We get

$$
\begin{aligned}
& \frac{m(B(x, r))}{r^{h}} \geq 4^{h} \frac{(a b)^{h}}{(b-a)^{h}}\left(\sum_{n=k}^{l-1} \frac{1_{I}(n)}{n^{2 h}}\right) \\
& \quad \geq 4^{h} \frac{(a b)^{h}}{(b-a)^{h}}\left(\sum_{n=a}^{b} \frac{1_{I}(n)}{n^{2 h}}-\frac{1}{(k-1)^{2 h}}-\frac{1}{(k)^{2 h}}-\frac{1}{(l+1)^{2 h}}-\frac{1}{(l+2)^{2 h}}\right) \\
& \quad \geq 4^{h} \frac{(a b)^{h}}{(b-a)^{h}}\left(\sum_{n=a}^{b} \frac{1_{I}(n)}{n^{2 h}}\right)-4^{1+h} \frac{(a b)^{h}}{(b-a)^{h}(k-1)^{2 h}} .
\end{aligned}
$$

Now,

$$
\frac{(a b)^{h}}{(b-a)^{h}(k-1)^{2 h}} \leq \frac{(a b)^{h}}{(b-a)^{h}(a-1)^{2 h}} \leq\left(\frac{a}{a-1}\right)^{h} \cdot \frac{b^{h}}{(b-a)^{h} a^{h}} \leq \frac{M}{8},
$$

provided that $k$ and $l-k$ are large enough (depending only on $M$ ) and then $m(B(x, r)) / r^{h} \geq 4^{-h} M / 2$. But $k$ will be as large as we wish by taking $i$ sufficiently large and since $l-k \geq r i^{2} \geq \frac{\gamma}{i+1} i \geq \gamma / 2, l-k$ will be as large as we wish choosing $\gamma$ large enough. Applying now Theorem 2.5(2) we finish the case when $r<1 / i$. In case $r \geq 1 / i$, set $k=\left[1 /\left(\frac{1}{i}+r\right)\right]+1$ as before. Then $1 / i+r \geq 1 / k$ and therefore taking $x \in J \cap(1 /(i+1), 1 / i)$ sufficiently close to $1 / i$, we get $B(x, r) \cap J \supset(0,1 /(k+1)) \cap J=\bigcup_{n \geq k+1}[1 /(n+1), 1 / n] \cap J$. Thus

$$
\frac{m(B(x, r))}{r^{h}} \geq \frac{K^{-h}}{r^{h}} \sum_{n=k+1}^{\infty} \frac{1_{I}(n)}{n^{2 h}} .
$$

Since $1 / r \geq \frac{1}{\frac{1}{i}+r} \geq k-1$,

$$
\frac{m(B(x, r))}{r^{h}} \geq K^{-h}(k-1)^{h} \sum_{n=k+1}^{\infty} \frac{1_{I}(n)}{n^{2 h}}=K^{-h}\left(\frac{k-1}{k+1}\right)^{h}(k+1)^{h} \sum_{n=k+1}^{\infty} \frac{1_{I}(n)}{n^{2 h}} .
$$

Taking $i$ large enough, we get $k \geq 2$ and $\frac{k-1}{k+1} \geq 1 / 3$. Since the second infimum in (b) is positive, the proof is completed.

Lemma 5.2. If $\mathcal{P}^{h}(J)<\infty$, then $h \leq 2 \theta$. 
Proof. Let $A>0$ be the first infimum appearing in Theorem 5.1(b). If $l \in I$ is large enough and $k=[2 l / 3]$, then $\left|\frac{2 k(2 l)}{k+2 l}-l\right|<1$. So,

$$
\frac{A}{2}<\frac{(k)^{h}(2 l)^{h}}{(k+2 l)^{h}} \sum_{n=k}^{2 l} \frac{1_{I}(n)}{n^{2 h}} \asymp l^{h} \frac{\# I(2 l / 3,2 l)}{l^{2 h}}=\frac{\# I(2 l / 3,2 l)}{l^{h}} .
$$

Since $I$ is infinite, there exists an infinite sequence $F \subset I$ such that $[2 l / 3,2 l] \cap$ $[2 s / 3,2 s]=\emptyset$ for all distinct elements $l$ and $s$ of $F$. Hence,

$$
\psi_{1}(h / 2) \asymp \sum_{l \in I} \frac{1}{l^{h}} \geq \sum_{l \in F} \sum_{n=2 l / 3}^{2 l} \frac{1}{n^{h}} \asymp \sum_{l \in F} \frac{1}{(2 l)^{h}} \# I(2 l / 3,2 l) \geq \frac{A}{2} \sum_{l \in F} 2^{-h}=\infty .
$$

Thus, $h / 2 \leq \theta$ and we are done.

Combining this lemma and Proposition 4.4 we get the following

Proposition 5.3. If $\mathcal{H}^{h}(J)>0$ and $\mathcal{P}^{h}(J)<\infty$, then $h=2 \theta$.

The proof of the following consequence of Lemma 5.2 is similar to the proof of Theorem 4.9 .

Theorem 5.4. Let $I \subset \mathbb{N}$ induce a regular continued fraction system and suppose $\theta<1 / 2$. Then there exists a number $q \geq 1$ such that if $F$ is strongly equivalent with $I$ and $F \supset[1, q]$, then $\mathcal{P}^{h}\left(J_{F}\right)=\infty$.

Proof. In view of Theorem 1.1, there exists $q \geq 1$ such that $\operatorname{dim}_{\mathrm{H}}\left(J_{F}\right)>2 \theta_{I}$ if $F \supset[1, q]$. Now applying Lemma 5.2 along with the fact that strongly equivalent sets have the same finiteness parameter, $\theta$, finishes the proof.

We shall now prove the following.

Lemma 5.5. If $\mathbb{N} \backslash I$ contains punctured clusters of arbitrarily large lower lengths, then $\mathcal{P}^{h}\left(J_{I}\right)=\infty$.

Proof. By assumption, $I$ contains an infinite sequence of triples $(a, n, b)$ and $a<$ $n<b, I \cap[a, b]=\{n\}$ such that $\min (b-n, n-a) \rightarrow \infty$. For each such triple, let $r=\inf \{s: 3 / 4 \leq s \leq 1, a \leq[s n],[r n / 2 r-1] \leq b-1\}$, let $k=[r n]$ and $l=[r n / 2 r-1]$. Then $k \rightarrow \infty$ and $l-k \rightarrow \infty$. Also, if $n$ is large enough, then $A\left(\frac{2 k l}{k+l}\right) \cap I \neq \emptyset$, and $[k, l] \cap I$ is a singleton contained in $A\left(\frac{2 k l}{k+l}\right)$. Therefore,

$$
\begin{aligned}
M_{k, l} & =\frac{k^{h} l^{h}}{(l-k)^{h}} \sum_{n=k}^{l} \frac{1_{I}(n)}{n^{2 h}} \asymp \frac{k^{h} l^{h}}{(l-k)^{h}}\left(\frac{k+l}{k l}\right)^{2 h} \asymp \frac{(k+l)^{2 h}}{(l-k)^{h}(k l)^{h}} \\
& \preceq 4^{h} \frac{l^{2 h}}{(l-k)^{h}(k l)^{h}}=4^{h} \frac{l^{h}}{k^{h}(l-k)^{h}} .
\end{aligned}
$$

Since $\max \{k, l-k\} \geq l / 2$ and since both numbers $k$ and $(l-k)$ diverge to $\infty$, it follows from this estimate that $M_{k, l} \rightarrow 0$ over such pairs of $k$ and $l$. Thus an application of Theorem 5.1 finishes the proof.

Corollary 5.6. Let I be the set of prime numbers. Then $\mathcal{P}^{h}\left(J_{I}\right)=\infty$.

Proof. It is known that the primes have arbitrarily large two sided gaps (see $[\mathrm{E}],[\mathrm{M}])$. The corollary follows.

Theorem 5.7. Let $I \subset \mathbb{N}$ be a proper infinite subset of $\mathbb{N}$. If $\# I(k, l) \succeq(l-k)^{h}$ for all $k<l$ with $A\left(\frac{2 k l}{k+l}\right) \cap I \neq \emptyset$, then the first infimum in Theorem $5.1(\bar{b})$ is positive. 
Proof. Consider $k<l$ such that $A\left(\frac{2 k l}{k+l}\right) \cap I \neq \emptyset$. Suppose first that $l \leq 2 k$. Then

$$
\frac{(k l)^{h}}{(l-k)^{h}} \sum_{n=k}^{l} \frac{1_{I}(n)}{n^{2 h}} \asymp \frac{k^{2 h}}{(l-k)^{h}} \# I(k, l) \frac{1}{k^{2 h}} \geq \frac{(l-k)^{h}}{(l-k)^{h}}=1 .
$$

If $l \geq 2 k$, then we can find $j$ such that $[k, l] \supset[j, 2 j]$ and $A\left(\frac{2 k l}{k+l}\right) \cap A\left(\frac{2 j 2 j}{j+2 j}\right) \neq \emptyset$. Since the points of $A\left(\frac{2 k l}{k+l}\right)$ are of order $k$ and $\frac{2 j 2 j}{j+2 j}=\frac{4}{3} j$, we see that the numbers $k$ and $j$ are of the same order. Hence

$$
\begin{aligned}
\frac{(k l)^{h}}{(l-k)^{h}} \sum_{n=k}^{l} \frac{1_{I}(n)}{n^{2 h}} & \asymp k^{h} \sum_{n=k}^{l} \frac{1_{I}(n)}{n^{2 h}} \succeq k^{h} \sum_{n=j}^{2 j} \frac{1_{I}(n)}{n^{2 h}} \\
& \succeq k^{h} \# I(j, 2 j) \cdot \frac{1}{j^{2 h}} \succeq k^{h} j^{h} j^{-2 h}=\left(\frac{k}{j}\right)^{h} \\
& \asymp 1 .
\end{aligned}
$$

This implies the first infimum in Theorem 5.1(b) is finite.

Lemma 5.8. If $\liminf _{n \rightarrow \infty} \frac{S_{n}}{n}>1-\frac{1}{2 h}$, then the second infimum in Theorem 5.1(b) is positive and $\theta=1 / 2$.

Proof. It is straightforward to check that $\psi(1 / 2)=\infty$. By summation by parts and the fact that $h>1 / 2$, we have

$$
k^{h} \sum_{n=k}^{\infty} \frac{1_{I}(n)}{n^{2 h}} \geq k^{h}\left[\sum_{n=k}^{\infty} \frac{2 h S_{n}}{n^{2 h+1}}-\frac{S_{k-1}}{k^{2 h}}\right] .
$$

Now, there exists $c>1-\frac{1}{2 h}$ such for all sufficiently large $k$,

$$
\begin{aligned}
k^{h} \sum_{n=k}^{\infty} \frac{1_{I}(n)}{n^{2 h}} & \geq(2 h) c k^{h}\left[\sum_{n=k}^{\infty} \frac{1}{n^{2 h}}\right]-\frac{S_{k-1}}{k^{h}} \\
& \geq k^{1-h}\left[\frac{2 h}{2 h-1} c-\frac{S_{k-1}}{k}\right] \geq k^{1-h}\left[\frac{2 h}{2 h-1} c-1\right] .
\end{aligned}
$$

Since $\frac{2 h}{2 h-1} c-1>0$, this implies the second infimum in Theorem 5.1(b) is positive.

Corollary 5.9. If $I \subset \mathbb{N}$ has bounded gaps, in particular, if I contains an infinite arithmetic progression, then $\mathcal{P}^{h}(J)<\infty$.

Proof. If $I$ has gaps bounded by $d$, then the lower arithmetic density of $I$ is $\geq 1 / d$. Also, we have \#I(k,l) $\frac{1}{d}(l-k)$ for $k$ and $l-k$ large enough. So, by Theorems 5.7 and 5.8, both infima in Theorem 5.1(c) are positive and $\mathcal{P}^{h}(J)<\infty$.

Remark. There are subsets I with bounded gaps and which do not contain an infinite arithmetic progression.

We shall now formulate a sufficient condition for the first infimum in Theorem $5.1(\mathrm{c})$ to be positive.

Proposition 5.10. Let $I=\left\{a_{n}: n \geq 1\right\}$ be a subsequence of positive integers such that if $a_{n}-1 \notin I$, then $I \cap\left[a_{n}, \infty\right) \supset\left[a_{n}, 2 a_{n}\right]$. Then with $h=1$ the first infimum in Theorem 5.1(c) is positive. 
Proof. Let us fix $k, l \in \mathbb{N}$ such that $A\left(\frac{2 k l}{k+l}\right) \cap I \neq \emptyset$ and $l-k \geq 10$. Choose $c \in A\left(\frac{2 k l}{k+l}\right) \cap I \neq \emptyset$ and then fix a cluster of the form $[a, 2 a]$ containing $c$. Let us explore several cases:

Case 1. $[k, l] \subset[a, 2 a]$. Then

$$
\frac{k l}{l-k} \sum_{n=k}^{l} \frac{1_{I}(n)}{n^{2}} \geq \frac{k l}{l-k} \frac{l-k}{l^{2}}=\frac{k}{l} \geq \frac{1}{2} .
$$

Case 2. $[a, 2 a] \subset[k, l]$. Since $a \leq c \leq 2 k$, we get

$$
\frac{k l}{l-k} \sum_{n=k}^{l} \frac{1_{I}(n)}{n^{2}} \geq \frac{k l}{l-k} \sum_{n=a}^{2 a} \frac{1}{n^{2}} \geq \frac{k l}{l-k} \frac{a}{4 a^{2}}=\frac{1}{4} \frac{k l}{(l-k) a} \geq \frac{1}{4} \frac{k}{a} \geq \frac{1}{8} .
$$

Case 3. $k \notin[a, 2 a]$ and $l \in[a, 2 a]$. Since $c \in A\left(\frac{2 k l}{k+l}\right)$ we get, $l-c \geq \frac{l(l-k)}{l+k}-1$. Since also $2 k \geq c \geq a \geq l / 2$, we get

$$
\begin{aligned}
\frac{k l}{l-k} \sum_{n=k}^{l} \frac{1_{I}(n)}{n^{2}} & \geq \frac{k l}{l-k} \sum_{n=a}^{l} \frac{1}{n^{2}} \geq \frac{k l}{l-k} \frac{l-a}{l^{2}} \\
& \geq \frac{k(l-c)}{l(l-k)} \geq \frac{k l(l-k)}{l(l-k)(l+k)}-\frac{k}{l(l-k)}=\frac{k}{l+k}-\frac{k}{l(l-k)} \\
& \geq \frac{1}{5}-\frac{1}{10} \\
& =\frac{1}{10} .
\end{aligned}
$$

Case 4. $k \in[a, 2 a]$ and $l \notin[a, 2 a]$. Since $\frac{k l}{l-k} \geq \frac{2 k(c+1)}{c+1-k}$, we get

$$
\begin{aligned}
\frac{k l}{l-k} \sum_{n=k}^{l} \frac{1_{I}(n)}{n^{2}} & \geq \frac{2 k(c+1)}{c+1-k} \sum_{n=k}^{2 a} \frac{1}{n^{2}} \geq \frac{2 k(c+1)}{c+1-k} \cdot \frac{2 a-k}{4 a^{2}} \\
& \geq \frac{2 a^{2}(2 a-k)}{4 a^{2}(c+1-k)}=\frac{1}{2} \frac{2 a-k}{c+1-k} \geq \frac{1}{2} \frac{c-k}{c+1-k} \\
& \geq \frac{1}{4} .
\end{aligned}
$$

The proof is finished.

Remark. By Lemma 4.11, the infimum considered in Proposition 5.10 is positive for all $0 \leq h \leq 1$.

We shall now construct two examples showing that in general the two inequalities in Theorem 5.1(c) are mutually independent.

Example 5.11. Here we construct an example of a regular system showing that the second infimum in Theorem 5.1(c) may happen to be zero although the first one is positive. It goes as follows. Fix $1 / 2<s<1$. We will define by induction an infinite sequence $\left\{a_{n}: n \geq 1\right\}$ such that for $I_{1}=\bigcup_{n \geq 1}\left[a_{n}, 2 a_{n}\right]$, the second infimum, taken with this $s$, in Theorem 5.1(c) fails to be positive. Indeed, set $a_{1}=1$ and suppose that $a_{n}$ is already defined. The first restriction on $a_{n+1}$ is that 
$a_{n+1}>2 a_{n}$. Then

$$
\begin{aligned}
\left(2 a_{n}+1\right)^{s} \sum_{j=2 a_{n}+1}^{\infty} \frac{1}{j^{2 s}} & \leq 3^{s} a_{n}^{s} \sum_{j=a_{n+1}}^{\infty} \frac{1}{j^{2 s}} \leq 3^{s} a_{n}^{s} \frac{1}{2 s-1} a_{n+1}^{1-2 s} . \\
& =\frac{3^{s} 2^{1-2 s}}{2 s-1} a_{n}^{s} a_{n+1}^{1-2 s}
\end{aligned}
$$

Since $1-2 s<0$, we can find $a_{n+1}>2 a_{n}$ so large that

$$
\frac{3^{s} 2^{1-2 s}}{2 s-1} a_{n}^{s} a_{n+1}^{1-2 s} \leq \frac{1}{n+1} .
$$

The construction of $I_{1}$ is finished. The first infimum in Theorem 5.1(c) is positive by Proposition 5.10 and the second one is zero by (5.1). We now define the set $I$ adding to $I_{1}$ an initial segment of the form $[1,2 p]$ so long that $\operatorname{dim}_{H}\left(J_{I}\right)>\max \left\{s, \theta_{I}\right\}$. Then $I$ induces a regular system (see Theorem 1.2), $I$ continuous to satisfy the assumptions of Proposition 5.10 and the second infimum in Theorem 5.1(c) is zero by (5.1), Lemma 4.11 and since $I \cap[2 p+1, \infty)=I_{1} \cap[2 p+1, \infty)$.

Example 5.12. We shall now describe a regular system for which the first infimum in Theorem 5.1(c) is zero but the second one is positive. Indeed, let $I=$ $\bigcup_{n \geq 0}\left(\left[4^{n}, 2 \cdot 4^{n}\right] \cup\left\{3 \cdot 2^{n}\right\}\right)$. Then the complement of $I$ contains arbitrarily long punctured clusters and therefore the first infimum in Theorem 5.1(c) is zero by Theorem 5.5. In order to check that the second infimum is positive, given $k \geq 1$, consider $n \geq 0$ such that $4^{n} \leq k \leq 4^{n+1}$. Then

$$
k \sum_{j=k}^{\infty} \frac{1_{I}(j)}{j^{2}} \geq 4^{n} \sum_{j=4^{n+1}}^{2 \cdot 4^{n+1}} \frac{1}{j^{2}} \geq 4^{n} \cdot 4^{n+1} \cdot \frac{1}{4 \cdot 4^{2 n+2}}=\frac{1}{16} .
$$

Since $\theta_{I}=1 / 2$ and $\psi_{I}(1 / 2)=\infty$, the system generated by $I$ is cofinitely regular. We are done.

Example 5.13. Consider $I=\bigcup_{n \geq 0}\left(\left[4^{n}, 2 \cdot 4^{n}\right] \cup\left\{3 \cdot 2^{n}\right\}\right)$. Then $I$ has positive arithmetic density, unbounded gaps and $\mathcal{P}^{h}\left(J_{I}\right)<\infty$. This is so, since the assumptions of Proposition 5.10 are satisfied and since the second infimum in Theorem 5.1(c) is positive which we check in exactly the same way as in Example 5.12. Notice that the sets $I$ considered here and in Example 5.12 differ only by a rather thin set $\left\{3 \cdot 2^{n}\right\}$ but the limit sets they generate have substantially different geometrical properties.

Theorem 5.14. If $\mathcal{P}^{h}\left(J_{I}\right)<\infty$, then $\lim \sup _{n \rightarrow \infty} \frac{S_{n}}{n^{h}}>0$.

Proof. By summation by parts,

$$
\sum_{n=k}^{l} \frac{1_{I}(n)}{n^{2 h}}=\sum_{n=k}^{l} S_{n}\left(\frac{1}{n^{2 h}}-\frac{1}{(n+1)^{2 h}}\right)+\frac{S_{l}}{(l+1)^{2 h}}-\frac{S_{k-1}}{(k)^{2 h}} .
$$

If $\lim _{\sup _{n \rightarrow \infty}} \frac{S_{n}}{n^{h}}=0$, then, for each $k$,

$$
k^{h} \sum_{n=k}^{\infty} \frac{1_{I}(n)}{n^{2 h}}=k^{h}\left[\sum_{n=k}^{\infty} S_{n}\left(\frac{1}{n^{2 h}}-\frac{1}{(n+1)^{2 h}}\right)-\frac{S_{k-1}}{k^{2 h}}\right] \preceq \sup _{n \geq k} \frac{S_{n}}{n^{h}}-\frac{S_{k-1}}{k^{h}} .
$$

But, the right-hand side converges to 0 as $k \rightarrow \infty$. Thus, the second infimum in Theorem 5.1(b) is not positive and we have a contradiction. 
Corollary 5.15. If I is the base for an absolutely regular system, then $\mathcal{P}^{h}\left(J_{I}\right)=\infty$.

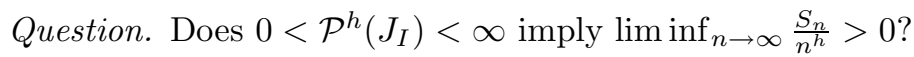

Lemma 5.16. Let $I \subset \mathbb{N}$. If, for some $s \geq 0$, we have $k^{s} \sum_{n=k}^{l} \frac{1_{I}(n)}{n^{2 s}}>L>0$, for all $k \geq 1$, then $s \leq 2 \theta$.

Proof. For all $k \geq 1$,

$$
\sum_{n \geq k} \frac{1_{I}(n)}{n^{s}} \geq \sum_{n \geq k} \frac{1_{I}(n) k^{s}}{n^{2 s}}=k^{s} \sum_{n \geq k} \frac{1_{I}(n)}{n^{2 s}} \geq L .
$$

Therefore, $\psi_{1}(s / 2)=\sum_{n=1}^{\infty} \frac{1_{I}(n)}{n^{s}}=\infty$. So, $\theta \geq s / 2$.

Theorem 5.17. If $I \subset \mathbb{N}$ and $\mathcal{P}^{h_{I}}\left(J_{I}\right)<\infty$, then $\mathcal{P}^{h_{E}}\left(J_{E}\right)<\infty$, for every cofinite regular subsystem $E$ of $I$.

Proof. Let $k_{0}$ be such that $E \cap\left[k_{0}, \infty\right)=I \cap\left[k_{0}, \infty\right)$. Using Lemma 4.11 we see that condition (c) of Theorem 5.1 is satisfied for the system with base E. Thus, $\mathcal{P}^{h_{E}}\left(J_{E}\right)<\infty$.

We finish this section with its most constructive theorem whose proof shows how to produce sets of arithmetic density zero, but whose limit sets have finite packing measure.

Theorem 5.18. There exist infinite sets $I \subset \mathbb{N}$ such that the induced continued fraction systems are strongly regular, $\mathcal{H}^{h}\left(J_{I}\right)=0, \mathcal{P}^{h}\left(J_{I}\right)<\infty$, and both numbers $\theta_{I}$ and $h_{I}$ are arbitrarily close to zero.

Proof. Fix an integer $p>4$ and $3<\alpha<p-1$. We will show that if the integer $w$ is large enough, then the systems generated by the sets of the form

$$
I=I(p, \alpha, w)=\bigcup_{n \geq w}\left[n^{p}+n^{\alpha}\right]
$$

satisfy the requirements of our theorem. We shall show first that the system generated by the set of entries $I=I(p, \alpha, w)$ is strongly regular and $\theta_{I(p, \alpha, w)}=\frac{1+\alpha}{2 p}$. Indeed, this follows from the following computation:

$$
\psi_{1}(t)=\sum_{n \geq w} \sum_{j=n^{p}}^{n^{p}+n^{\alpha}} \frac{1}{j^{2 t}} \asymp \sum_{n \geq w} \frac{1}{n^{2 p t}} n^{\alpha}=\sum_{n \geq w} \frac{1}{n^{2 p t-\alpha}} .
$$

Let, as usual, $h$ denote the Hausdorff dimension of $J_{I}$, the limit set generated by the set $I(p, \alpha, w)$. By Lemma 4.7, $\mathcal{H}^{h}\left(J_{I}\right)=0$. In order to prove that $\mathcal{P}^{h}\left(J_{I}\right)<\infty$, we shall demonstrate that the assumptions of Theorem 5.1(c) are satisfied. Indeed, in order to verify that the second infimum in Theorem 5.1(c) is positive it suffices to check that $\liminf _{k \rightarrow \infty} M_{k}>0$, where $M_{k}=k^{p h} \sum_{n \geq k} \sum_{j=n^{p}}^{n^{p}+n^{\alpha}} j^{-2 h}$. We do it as follows.

$$
\begin{aligned}
M_{k} & =k^{p h} \sum_{n \geq k} \sum_{j=n^{p}}^{n^{p}+n^{\alpha}} j^{-2 h} \asymp k^{p h} \sum_{n \geq k} n^{-2 p h} n^{\alpha}=k^{p h} \sum_{n \geq k} n^{\alpha-2 p h} \\
& \asymp k^{p h} \int_{k}^{\infty} x^{\alpha-2 p h} d x=\frac{k^{p h}}{\alpha-2 p h+1}\left[x^{\alpha-2 p h+1}\right]_{k}^{\infty} .
\end{aligned}
$$


Notice now that since $h>\theta_{I}=\frac{1+\alpha}{2 p}$, we have $\alpha-2 p h+1<0$, and therefore $M_{k} \asymp k^{\alpha-p h+1}$. Hence

$$
\liminf _{k \rightarrow \infty} M_{k}>0 \Leftrightarrow \alpha \geq p h+1 .
$$

In order to check that the first condition of Theorem 5.1(c) is satisfied, set

$$
M_{k, l}=\frac{(k l)^{h}}{(l-k)^{h}} \sum_{n=k}^{l} n^{-2 h} \mathbb{1}_{I(p, w, \alpha)}(n) .
$$

We want to show that $M_{k, l}$ with $A\left(\frac{2 k l}{k+l}\right) \cap I(p, w, \alpha) \neq \emptyset$ are bounded away from zero. Let $n$ be the only integer with $(n-1)^{p} \leq k<n^{p}$, and let $m$ be the only integer with $m^{p} \leq l<(m+1)^{p}$. We shall consider several cases:

Case 1. $m \geq n+2$. Then we may estimate the number $M_{k, l}$ from below as follows.

$$
\begin{aligned}
M_{k, l} & \geq \frac{(k l)^{h}}{(l-k)^{h}} \sum_{j=n}^{m-1} \sum_{s=j^{p}}^{j^{p}+j^{\alpha}} \frac{1}{s^{2 h}} \asymp \frac{(k l)^{h}}{(l-k)^{h}} \sum_{j=n}^{m-1} \frac{1}{j^{2 p h}} j^{\alpha} \\
& =\frac{(k l)^{h}}{(l-k) h} \sum_{j=n}^{m-1} j^{\alpha-2 p h} \asymp \frac{(k l)^{h}}{(l-k)^{h}} \int_{n}^{m-1} x^{\alpha-2 p h} d x \\
& \asymp \frac{(k l)^{h}}{(l-k)^{h}} \frac{1}{\alpha-2 p h+1}\left((m-1)^{\alpha-2 p h+1}-n^{\alpha-2 p h+1}\right) \\
& \succeq \frac{(n m)^{p h}}{\left((m+1)^{p}-(n-1)^{p}\right)^{h}}\left((m-1)^{\alpha-2 p h+1}-n^{\alpha-2 p h+1}\right) \\
& =\frac{(n m)^{p h}}{\left((m+1)^{p}-(n-1)^{p}\right)^{h}}\left((m-1)^{p}-n^{p}\right) x^{\frac{1+\alpha-2 p h}{p}-1} .
\end{aligned}
$$

for some $x$, where $n^{p} \leq x \leq(m-1)^{p}$. We continue the above estimates as follows.

$$
M_{k, l} \geq \frac{1}{2}(n m)^{p h} x^{\frac{1+\alpha-2 p h-p}{p}}\left(m^{p}-n^{p}\right)^{1-h} .
$$

Since $1+\alpha-2 p h-p<0$, we get

$$
\begin{aligned}
M_{k, l} & \succeq(n m)^{p h} m^{\frac{1+\alpha-2 p h-p}{p}}\left(m^{p}-n^{p}\right)^{1-h} \\
& \succeq n^{p h}\left(m^{p}-n^{p}\right)^{1-h} m^{\frac{1+\alpha-2 p h+p^{2} h-p}{p}} .
\end{aligned}
$$

Thus, if

$$
1+\alpha-2 p h+p^{2} h-p \geq 0,
$$

then the quantities $M_{k, l}$ with $m \geq n+2$ are bounded away from zero.

Case 2a. We now assume that $(n-1)^{p} \leq k \leq n^{p}$ and $(n+1)^{p}+(n+1)^{\alpha} \leq l<$ $(n+2)^{p}$. Then

$$
\begin{aligned}
M_{k, l} & \succeq\left(\frac{k l}{l-k}\right)^{h} \frac{(n+1)^{\alpha}}{n^{2 p h}} \succeq \frac{n^{2 p h}}{(l-k)^{h}} \cdot \frac{n^{\alpha}}{n^{2 p h}} \\
& =\frac{n^{\alpha}}{(l-k)^{h}} \succeq \frac{n^{\alpha}}{\left((n+2)^{p}-(n-1)^{p}\right)^{h}} \asymp \frac{n^{\alpha}}{n^{(p-1) h}} .
\end{aligned}
$$

So, if

$$
\alpha-(p-1) h \leq 0,
$$

then $M_{k, l}$ is bounded away from 0 . 
Case 2b. $(n-1)^{p} \leq k<n^{p}$ and $(n+1)^{p} \leq l<(n+1)^{p}+(n+1)^{\alpha}$. We shall show that in this case if $n$ is large enough, then $A\left(\frac{2 k l}{k+l}\right) \cap I=\emptyset$. And indeed, for this intersection to be empty it suffices to know that

$$
n^{p}+n^{\alpha}<\frac{2 k l}{k+l}-1 \text { and } \frac{2 k l}{k+l}+1<(n+1)^{p} .
$$

But the harmonic mean $\frac{2 k l}{k+l}$ takes on its minimum if $k$ and $l$ are minimal and it takes on its maximum if $k$ and $l$ are maximal. Thus our task reduces to check that

$$
n^{p}+n^{\alpha}<\frac{2\left(n_{1}\right)^{p}(n+1)^{p}}{(n-1)^{p}+(n+1)^{p}}-1 \text { and } \frac{2 n^{p}\left((n+1)^{p}+(n+1)^{\alpha}\right)}{n^{p}+(n+1)^{p}+(n+1)^{\alpha}}<(n+1)^{p}
$$

provided $n$ is large enough. This can be verified by a straightforward computation.

Case 3. $(n-1)^{p} \leq k<n^{p}$ and $n^{p} \leq l<(n+1)^{p}$. We shall consider three subcases.

Case 3a. $n^{p}+n^{\alpha} \leq l<(n+1)^{p}$. Then

$$
\begin{aligned}
M_{k, l} & \succeq\left(\frac{k l}{l-k}\right)^{h} \cdot n^{\alpha} \cdot \frac{1}{n^{2 p h}} \succeq \frac{n^{2 p h}}{(l-k)^{h}} \cdot n^{\alpha} \cdot \frac{1}{n^{2 p h}} \\
& =\frac{n^{\alpha}}{(l-k)^{h}} \succeq \frac{n^{\alpha}}{\left((n+1)^{p}-(n-1)^{p}\right)^{h}} \asymp \frac{n^{\alpha}}{n^{(p-1) h}} \\
& =n^{\alpha-(p-1) h} .
\end{aligned}
$$

So, again if $\alpha-(p-1) h \geq 0$, then we are done.

Case 3b. $n^{p} \leq l<n^{p}+n^{\alpha}$ and $(n-1)^{p}+(n-1)^{\alpha} \leq k$. Then

$$
M_{k, l} \succeq\left(\frac{k l}{l-k}\right)^{h} \frac{1}{l^{2 h}}\left(l-n^{p}+1\right) \succeq \frac{l-n^{p}}{(l-k)^{h}} .
$$

But since $A\left(\frac{2 k l}{k+l}\right) \cap I \neq \emptyset$, we conclude that $\frac{2 k l}{k+l} \geq n^{p}-1$. So, $l-n^{p} \geq l-q-1$. But since $l-\frac{2 k l}{k+l} \geq(l-k) / 3$, we get

$$
M_{k, l} \succeq \frac{\frac{1}{3}(l-k)-1}{(l-k)^{h}} \asymp(l-k)^{1-h} \succeq 1 .
$$

Case 3c. $n^{p} \leq l<n^{p}+n^{\alpha}$ and $(n-1)^{p} \leq k<(n-1)^{p}+(n-1)^{\alpha}$. Then for all $n$ large enough

$$
2 n^{p}(n-1) 6 p>\left(n^{p}+(n-1)^{p}\right)\left((n-1)^{p}+(n-1)^{\alpha}+1\right)
$$

or

$$
\frac{2 n^{p}(n-1)^{p}}{n^{p}+(n-1)^{p}}>(n-1)^{p}+(n-1)^{\alpha}+1
$$

So,

$$
\frac{2 k l}{k+l} \geq \frac{2 n^{p}(n-1)^{p}}{n^{p}+(n-1)^{p}}>(n-1)^{p}+(n-1)^{\alpha}+1
$$

and consequently $A\left(\frac{2 k l}{k+l}\right) \geq n^{p}$. But then

$$
M_{k, l} \asymp\left(\frac{k l}{l-k}\right)^{h} \cdot \frac{1}{k^{h}}(l-q)^{h} \asymp\left(\frac{l-q}{l-k}\right)^{h} \geq\left(\frac{1}{3}\right)^{h}(l-k)^{h} \succeq 1,
$$

and we are done in this case. 
Case 4. $(n-1)^{p} \leq k<l<n^{p}$. In order for $A\left(\frac{2 k l}{k+l} \cap I \neq \emptyset,(n-1)^{p} \leq k \leq\right.$ $(n-1)^{p}+(n-1)^{\alpha}$.

Case 4a. $l \leq(n-1)^{p}+(n-1)^{\alpha}$. Then

$$
M_{k, l} \asymp\left(\frac{k l}{l-k}\right)^{h} \cdot \frac{1}{l^{2 h}}(l-k) \succeq(l-k)^{1-h} \geq 1,
$$

and we are done.

Case 4b. $(n-1)^{p}+(n-1)^{\alpha}<l$. Let $q=\frac{2 k l}{k+l}$. We then get

$$
\begin{aligned}
M_{k, l} & \succeq\left(\frac{k l}{l-k}\right)^{h} \cdot \frac{1}{\left((n-1)^{p}+(n-1)^{\alpha}\right)^{2 h}}\left((n-1)^{p}+(n-1)^{\alpha}-k\right) \\
& \succeq \frac{(n-1)^{p}+(n-1)^{\alpha}-k}{(l-k)^{h}} \geq \frac{q-k}{(l-k)^{h}}
\end{aligned}
$$

Now, $q-k \geq(l-k) / 3$, so $M_{k, l} \succeq(l-k)^{1-h} \geq 1$ and we have finished this last case.

Since $\alpha>p \theta+1$ and $h \rightarrow \theta$ as $w \rightarrow \infty$, if $w$ is large enough $\alpha>p h+1$. The proof is completed.

\section{Dimension RELATIOns And FURTHER EXAMPles}

In this section, we give some examples of strongly regular systems with $\operatorname{dim}_{\mathrm{H}}(J)$ $<\operatorname{dim}_{\mathrm{P}}(J)$ and some examples with equality of these two dimensions.

Theorem 6.1. Let $I_{p}=\left\{n^{p}: n \geq 1\right\}$. If $p \geq 2$, then $\operatorname{dim}_{P}\left(J_{I_{p}}\right)=h_{I_{p}}>$ $1 / p, \quad \mathcal{H}^{h}\left(J_{I_{p}}\right)>0$ and $\mathcal{P}^{h}\left(J_{I_{p}}\right)=\infty$.

Proof. Since $\mathbb{N} \backslash I_{p}$ has punctured clusters of arbitrarily long lower lengths, $\mathcal{P}^{h}\left(J_{I_{p}}\right)$ $=\infty$ follows from Lemma 5.5. We will show that $h>1 / p$ by showing that $P(1 / p)>$ 0 . Since it is easy to calculate that $\operatorname{dim}_{B}\left(L_{1}(0)\right)=1 /(p+1)$, it would then follow from Theorem 2.11 that the box and Hausdorff dimensions of $J_{I_{p}}$ are equal. First, we estimate $\psi_{n+1}(t)$ from below as follows:

$$
\begin{aligned}
\psi_{n+1}(t) & =\sum_{\omega \in I^{n+1}}\left\|\phi_{\omega}^{\prime}\right\|^{t}=\sum_{\omega \in I^{n+1}} \frac{1}{\left(q_{n+1}(\omega)\right)^{2 t}}=\sum_{\omega \in I^{n}} \sum_{b \in I} \frac{1}{\left(b q_{n}+q_{n-1}\right)^{2 t}} \\
& =\sum_{\omega \in I^{n}} \frac{1}{q_{n}^{2 t}}\left[\sum_{b \in I} \frac{1}{\left(b+q_{n-1} / q_{n}\right)^{2 t}}\right] \geq \psi_{n}(t) \sum_{b \in I} \frac{1}{(b+1)^{2 t}} .
\end{aligned}
$$

Therefore, by induction we have

$$
\psi_{n}(t) \geq\left(\sum_{b \in I} \frac{1}{b^{2 t}}\right)\left(\sum_{b \in I} \frac{1}{(b+1)^{2 t}}\right)^{n-1} .
$$

So, if $\sum_{b \in I} \frac{1}{(b+1)^{2 t}}>1$, then $P(t)>0$. It can be checked that $\sum_{k=1}^{\infty}\left(\frac{1}{k^{p}+1}\right)^{2 / p}>1$, for all $p \geq 2$. Finally, since $\varrho \bar{\varrho}(I)=1 / p$, it follows from Lemma 4.8 that $\mathcal{H}^{h}\left(J_{I_{p}}\right)>$ 0 .

Theorem 6.2. For every $p \geq 2$, there exists $q \geq 1$ such that if $l \geq q$ and $I_{l}=\left\{n^{p}\right.$ : $n \geq l\}$, then $\operatorname{dim}_{H}\left(J_{l}\right)<\underline{\operatorname{dim}}_{B}\left(J_{l}\right) \leq \overline{\operatorname{dim}}_{B}\left(J_{l}\right)=\operatorname{dim}_{P}\left(J_{l}\right)$. 
Proof. First notice that for every $l \geq 1, \theta_{I_{l}}=1 / 2 p$ and $I_{l}$ is a regular subset of $\mathbb{N}$. According to Theorem 1.5, $\lim _{l \rightarrow \infty} \operatorname{dim}_{\mathrm{H}}\left(J_{l}\right)=1 / 2 p$ and since $1 / 2 p<1 /(p+1)$, there exists $q \geq 1$ so large that $\operatorname{dim}_{\mathrm{H}}\left(J_{l}\right)<\underline{\operatorname{dim}}_{\mathrm{B}}\left(J_{l}\right)$ for all $l \geq q$. The last two equality signs in Theorem 6.2 are consequences of Theorem 3.1 in [MU] and Theorem 2.11.

Remark. Notice that in contrast to the case $p \geq 2$, for $p=1$ and every system strongly equivalent with $I_{1}$, we have $\operatorname{dim}_{\mathrm{H}}\left(J_{l}\right)=\overline{\operatorname{dim}}_{\mathrm{B}}\left(J_{l}\right)=\operatorname{dim}_{\mathrm{P}}\left(J_{l}\right)$. This follows from Corollary 5.9 and Theorem 3.1 in [MU].

Theorem 6.3. If $S=\left\{\phi_{i}: i \in I\right\}$ is a conformal iterated function system and the index set $I$ is infinite, then for every $0<t<\theta$ there exists a set $I_{t} \subset I$ such that $\operatorname{dim}_{H}\left(J_{I_{t}}\right)=t$.

Proof. Without losing generality we may assume that $I=\mathbb{N}$. First we shall show that for every set $E \subset \mathbb{N}$ such that $\mathbb{N} \backslash E$ is infinite and for every $\varepsilon>0$ there exists $k \in \mathbb{N} \backslash E$ such that $\operatorname{dim}_{\mathrm{H}}\left(J_{E \cup\{k\}}\right) \leq \operatorname{dim}_{\mathrm{H}}\left(J_{E}\right)+\varepsilon$. Indeed, let $h=\operatorname{dim}_{\mathrm{H}}\left(J_{E}\right)$. By Theorem 1.2, $P_{E}(h+\varepsilon)<0$ and by the definition of pressure there exists $0<a<1$ and $j_{0} \geq 1$ such that $\psi_{E, j}(h+\varepsilon)<a^{j}$, if $j>j_{0}$. But, for every $k \in \mathbb{N} \backslash E$, we have

$$
\begin{aligned}
\psi_{E \cup\{k\}, n}(h+\varepsilon) \leq \sum_{j=0}^{n}\left(\begin{array}{c}
n \\
j
\end{array}\right) \psi_{E, j}(h+\varepsilon)\left\|\phi_{k}^{\prime}\right\|^{(n-j)(h+\varepsilon)} K^{(n-j)(h+\varepsilon)} \\
\leq\left[\sum_{j=0}^{j_{0}}\left(\begin{array}{c}
n \\
j
\end{array}\right) \psi_{E, j}(h+\varepsilon) K^{(n-j)(h+\varepsilon)}\right]\left\|\phi_{k}^{\prime}\right\|^{\left(n-j_{0}\right)(h+\varepsilon)}+\left(a+\left(K\left\|\phi_{k}^{\prime}\right\|\right)^{(h+\varepsilon)}\right)^{n} \\
\leq j_{0} \sup _{0 \leq j \leq}\left\{\psi_{E, j}(h+\varepsilon)\right\} n^{j_{0}} K^{n(h+\varepsilon)}\left\|\phi_{k}^{\prime}\right\|^{\left(n-j_{0}\right)(h+\varepsilon)}+\left(a+\left(K\left\|\phi_{k}^{\prime}\right\|\right)^{(h+\varepsilon)}\right)^{n} .
\end{aligned}
$$

Since $\left\|\phi_{k}^{\prime}\right\|$ is sufficiently small for $k$ sufficiently large, we have $\psi_{E \cup\{k\}, n}(h+\varepsilon)<$ 1 for all $n$ large enough. This implies $P_{E \cup\{k\}}(h+\varepsilon)<0$ and consequently $\operatorname{dim}_{\mathrm{H}}\left(J_{E \cup\{k\}}\right) \leq h+\varepsilon$. The claim is proved.

Passing to the actual proof, fix $0<t<\theta_{\mathbb{N}}$. We shall build the set $I_{t}$ by constructing inductively an increasing sequence $I_{n}$ of finite subsets of $I$ satisfying $\operatorname{dim}_{\mathrm{H}}\left(J_{I_{n}}\right)<t$ for all $n \geq 1$. We then will show that setting $I_{t}=\bigcup_{n \geq 1} I_{n}$ we have $\operatorname{dim}_{\mathrm{H}}\left(J_{I_{t}}\right)=t$. Indeed, let $I_{1}=\{1\}$ and suppose that $I_{n}$ is constructed and $\operatorname{dim}_{\mathrm{H}}\left(J_{I_{n}}\right)<t$. By the claim proved above there exists $k>\max \left\{I_{n}\right\}$ such that $\operatorname{dim}_{\mathrm{H}}\left(J_{I_{n} \cup\{k\}}\right)<t$. Let $k_{n+1}$ be such minimal $k$ and let $I_{n+1}=I_{n} \cup\left\{k_{n+1}\right\}$. The inductive construction is finished. Let $I_{t}=\bigcup_{n \geq 1} I_{n}$. This set is infinite. By Theorem $1.2 \operatorname{dim}_{\mathrm{H}}\left(J_{I_{t}}\right) \leq t$. If the set $\mathbb{N} \backslash I_{t}$ were finite, then because of Theorem $1.3 \operatorname{dim}_{\mathrm{H}}\left(J_{I_{t}}\right) \geq \theta_{\mathbb{N}} \geq t$, and we would have a contradiction. Thus, $\mathbb{N} \backslash I_{t}$ is infinite. If $\operatorname{dim}_{\mathrm{H}}\left(J_{I_{t}}\right)=t$, we are done. Otherwise, due to our claim we can find an element $q \in \mathbb{N} \backslash I_{t}$ such that $k_{n+1}>q>k_{n}$ and $\operatorname{dim}_{\mathrm{H}}\left(J_{I_{n} \cup\{q\}}\right)<t$. But then $\operatorname{dim}_{\mathrm{H}}\left(J_{I_{n} \cup\{q\}}\right) \leq \operatorname{dim}_{\mathrm{H}}\left(J_{I_{t} \cup\{q\}}\right)<t$ which contradicts the choice of $k_{n+1}$ and finishes the proof of our theorem.

In general Theorem 6.3 fails to be true for $t>\theta$. Indeed, below we provide an example.

Example 6.4. Consider a system of similarity maps on the interval $[0,1]$ given by two generators $\phi$ and $\psi$ with contraction coefficients $1 / 4$ and the maps $\phi_{n}$ with contraction coefficients $c^{n}$, where $c$ is so small that the sets $\phi([0,1]), \psi([0,1])$, and $\phi_{n}([0,1]), n \geq 1$, are mutually disjoint. Then $\operatorname{dim}_{\mathrm{H}}\left(J_{\{\phi, \psi\}}\right)=1 / 2$ but the Hausdorff 
dimension of any subsystem missing either $\phi$ or $\psi$ is bounded from above by the solution to the equation $(1 / 4)^{t}+c^{t} /\left(1-c^{t}\right)=0$. But $t=t(c)$, the solution to this equation converges to 0 if $c \rightarrow 0$. Therefore if $c$ is taken so small that $t=t(c)<1 / 4$, we have a gap of Hausdorff dimension between $t(c)$ and $1 / 4$.

Example 6.5. We give an example of an irregular continued fraction system. First notice that if $I \subset \mathbb{N}$ is an index set, we may obtain upper bounds on the the functions $\psi_{n}(t)$ by a similar method to that given in Example 6.1 for obtaining lower bounds. Thus, using the Bounded Distortion Property with $K=4$ and using the facts that $b<b+q_{n-1} / q_{n}<b+1$ and $b+1 \leq 2 b$, we have

$$
\left(\sum_{b \in I} \frac{1}{b^{2 t}}\right)^{n} \geq \psi_{n}(t) \geq 4^{-(n-1) t}\left(\sum_{b \in I} \frac{1}{b^{2 t}}\right)^{n} .
$$

From this we have

$$
\log \left(\sum_{b \in I} \frac{1}{b^{2 t}}\right) \geq P_{I}(t) \geq-t \log 4+\log \left(\sum_{b \in I} \frac{1}{b^{2 t}}\right) .
$$

In particular, if $p>1 / 2$ and we set $I=\left\{\left[n(\log n)^{p}\right]: n \geq n_{0}\right\}$, then $\sum_{b \in I} \frac{1}{b}<1$, provided $n_{0}$ is large enough and $\sum_{b \in I} \frac{1}{b^{s}}=\infty$, if $s<1$. Thus, $P_{I}(1 / 2)<0$ and $P_{I}(t)=\infty$ if $t<1 / 2$. So, this system is irregular.

\section{SOME PROBLEMS}

1. Is there a nontrivial subset $I$ of $\mathbb{N}$ such that $0<\mathcal{H}^{h}\left(J_{I}\right)$ and $\mathcal{P}^{h}\left(J_{I}\right)<\infty$ ? If there is such an $I$, we know that $0<\limsup _{n \rightarrow \infty} \frac{S_{n}}{n^{h}}<\infty$.

2. Is there a Hausdorff gauge function $g$ of the form $g(t)=t^{h} L(t)$, where $L$ is a slowly varying function such that $0<\mathcal{H}^{h}\left(J_{I}\right)$ or $\mathcal{P}^{h}\left(J_{I}\right)<\infty$, where $I$ is the set of prime numbers? Since some detailed information is known about the distribution of the two sided gaps in the primes, one can at least determine a class of $g$ for which these measures are either 0 or $\infty$.

3. By Theorem 6.3 we know that for every $0<t<1 / 2$ there exists a continued fraction system whose limit set has dimension $t$. We conjecture that this remains true for all $t \in(0,1]$.

\section{REFERENCES}

[Cu] T.W. Cusick, Hausdorff dimension of sets of continued fractions, Quart. J. Math. Oxford (2), 41 (1990), 277-286. MR 91m:11057

[E] P. Erdös, On the difference of consecutive primes, Quart. J. Oxford 6 (1935), 124-128.

[Go] I.J. Good, The fractional dimension theory of continued fractions, Proc. Camb. Philos. Soc. 37(1941), 199-228; Corrigenda, Math. Proc. Camb. Phil. Soc. 105(1989), 607; Addenda, J. Statist. Comput. and Simul. 32, Nos. 1-2 (1989), 64-68. MR 3:7b; MR 90i:28013

[He] D. Hensley, Continued fraction Cantor sets, Hausdorff dimension, and functional analysis, J. Number Th. 40(1992), 336-358. MR 93c:11058

[M] H. Maier, Chains of large gaps between consecutive primes, Advances Math. 39 (1981), 257-269. MR 82g:10061

[Ma] P. Mattila, Geometry of sets and measures in euclidean spaces, Cambridge Studies in Advanced Mathematics 44(Cambridge University Press, 1995). MR 96h:28006 
[MU] R.D. Mauldin, M. Urbański, Dimensions and measures in infinite iterated function systems, Proceedings London Math. Soc., (3) 73(1996), 105-154. MR 97c:28020

[Ra] G. Ramharter, Some metrical properties of continued fractions, Mathematika 30 (1983), 117-132. MR 85c: 11058

Department of Mathematics, University of North Texas, Denton, Texas 76203

E-mail address: mauldin@unt.edu

E-mail address: urbanski@unt.edu 